BULLETIN (New Series) OF THE

AMERICAN MATHEMATICAL SOCIETY

Volume 37, Number 3, Pages 309-336

S 0273-0979(00)00863-6

Article electronically published on February 24, 2000

\title{
LOCAL GEOMETRIC PROPERTIES OF REAL SUBMANIFOLDS IN COMPLEX SPACE
}

\author{
M. S. BAOUENDI, P. EBENFELT, AND LINDA PREISS ROTHSCHILD
}

\begin{abstract}
We survey some recent results on local geometric properties of real submanifolds of complex space. Our main focus is on the structure and properties of mappings between such submanifolds. We relate these results to the classification of real submanifolds under biholomorphic, algebraic, or formal transformations. Examples and open problems in this context are also mentioned.
\end{abstract}

\section{INTRODUCTION}

A fundamental theorem in the theory of one complex variable is the Riemann mapping theorem, which asserts that any proper simply connected domain $\Omega$ in the complex plane is biholomorphically equivalent to the unit disk; i.e. there exists an invertible holomorphic mapping sending $\Omega$ onto the unit disk. Moreover, it is a classical result (see Kellogg [Ke1912] ) that if $\Omega$ has a smooth $\left(C^{\infty}\right)$ boundary, then the mapping extends smoothly to the boundary $\partial \Omega$. Similarly, if $\partial \Omega$ is realanalytic, then the mapping extends holomorphically to an open neighborhood of $\bar{\Omega}$, the closure of $\Omega$. The classification (up to biholomorphic equivalence) of domains in $\mathbb{C}^{N}, N \geq 2$, is much more complicated. Already early in the 20th century, Poincaré [Po1907] showed that the bidisk is not biholomorphically equivalent to the unit ball in $\mathbb{C}^{2}$, even though both are bounded domains which are diffeomorphic to each other (and even real analytically equivalent). It was also known to him that two domains, chosen at random in a class of bounded diffeomorphic domains, are very unlikely to be biholomorphically equivalent.

A bounded domain $\Omega \subset \mathbb{C}^{N}$ with smooth boundary is called strictly pseudoconvex if, at every point $p \in \partial \Omega$, there exists a local biholomorphism defined near $p$ which sends a neighborhood of $p$ in $\partial \Omega$ into a strictly convex hypersurface $\Sigma \subset \mathbb{C}^{N} \cong \mathbb{R}^{2 N}$ such that a neighborhood of $p$ in $\bar{\Omega}$ is mapped to the convex side of $\Sigma$. (Here, strictly convex means that $\Sigma$ is graphed over its tangent plane by a function with positive definite Hessian.) A result of Fefferman [Fe1974] states that a biholomorphic mapping $H$ between two smoothly bounded, strictly pseudoconvex domains $\Omega, \Omega^{\prime} \subset \mathbb{C}^{N}$ extends smoothly as a diffeomorphism between their boundaries $\partial \Omega$ and $\partial \Omega^{\prime}$. It follows from the work of Pinchuk Pi1975] and Lewy [Le1977]

Received by the editors July 22, 1999, and in revised form October 28, 1999.

2000 Mathematics Subject Classification. Primary 32V40, 32V35, 32V25, 32H02, 32V15, $32 \mathrm{~T} 15$.

The first and the third authors are partially supported by National Science Foundation grant DMS 98-01258. The second author is supported by a grant from the Swedish Natural Science Research Council.

(C)2000 American Mathematical Society 
that if $\partial \Omega$ and $\partial \Omega^{\prime}$ are real-analytic, then $H$ extends as a biholomorphism between open neighborhoods of $\bar{\Omega}$ and $\bar{\Omega}^{\prime}$, sending $\partial \Omega$ onto $\partial \Omega^{\prime}$. These results relate the biholomorphic classification of strictly pseudoconvex domains to the local invariant geometry of their boundaries (see also §1). E. Cartan Ca1932a, Ca1932b obtained the local biholomorphic classification of strictly pseudoconvex hypersurfaces in $\mathbb{C}^{2}$. The classification in higher dimensions was obtained by Chern and Moser CM1974 (see also earlier work by Tanaka Ta1962, Ta1967]). (The holomorphic extension across the boundary of mappings between real-analytic strictly pseudoconvex domains mentioned above also follows from the work in [CM1974].) This work is discussed in more detail in $\S 2$.

In this survey, we shall mostly focus on local problems. Let $M, M^{\prime} \subset \mathbb{C}^{N}$ be real submanifolds with $\operatorname{dim} M=\operatorname{dim} M^{\prime}$ and distinguished points $p_{0} \in M, p_{0}^{\prime} \in$ $M^{\prime}$. The local biholomorphic equivalence problem is to determine when $\left(M, p_{0}\right)$ and $\left(M^{\prime}, p_{0}^{\prime}\right)$ are biholomorphically equivalent, i.e. when there exists a germ at $p_{0}$ of a biholomorphism sending $M$ into $M^{\prime}$ and $p_{0}$ to $p_{0}^{\prime}$. In general, one would expect that $\left(M, p_{0}\right)$ and $\left(M^{\prime}, p_{0}^{\prime}\right)$ are not equivalent. In fact, the "probability" that $\left(M, p_{0}\right)$ and $\left(M^{\prime}, p_{0}^{\prime}\right)$, chosen at random, are biholomorphically equivalent is zero. An elementary argument (due to Poincaré Po1907]) supporting this statement will be given at the end of $\S 4$.

The local biholomorphic equivalence problem, in the classical context of normal forms and invariants, is addressed for Levi nondegenerate hypersurfaces in $\S 2$. For $N$ dimensional real submanifolds in $\mathbb{C}^{N}$ with complex tangents, it is addressed in $\S 3$ following the work of Moser and Webster [MW1983].

An important invariant object attached to $\left(M, p_{0}\right)$ is the group (under composition) of germs at $p_{0}$ of biholomorphisms mapping $M$ into itself and preserving $p_{0}$. This group is called the stability group of $M$ at $p_{0}$ and is denoted by $\operatorname{Aut}\left(M, p_{0}\right)$. Conditions on $\left(M, p_{0}\right)$ under which the stability group is a finite dimensional Lie group are given in $\S 4$ of this survey.

Another aspect of the local equivalence problem is the following. Suppose that there is a formal equivalence (in the sense of formal power series, see $\S 7$ ) between $\left(M, p_{0}\right)$ and $\left(M^{\prime}, p_{0}^{\prime}\right)$. Are they then biholomorphically equivalent? In particular, does the given formal equivalence converge? This problem is addressed in $\S \S 2-3$ and $\$ \S 6-7$ in this survey. A stronger equivalence problem is that of algebraic equivalence when $M$ and $M^{\prime}$ are real algebraic submanifolds. In particular, assuming that $\left(M, p_{0}\right)$ and $\left(M^{\prime}, p_{0}^{\prime}\right)$ are biholomorphically equivalent, are they then algebraically equivalent? As above, one may also ask whether the given biholomorphic equivalence is in fact algebraic. Some results are given in $\S \S 5-6$.

The study of real submanifolds in complex space is a very active area of research, and there is a vast literature related to the subject. In this survey we have chosen to mention only a few selected topics, and this choice is based purely on the authors' current interests and prejudices. We have made a special effort to mention more recent results, but we have left many areas untouched.

We would like to thank X. Huang for many useful remarks and suggestions concerning several topics in this survey. We thank X. Gong for his help with references and historical comments, and also A. Isaev, S. Ji, N. Stanton, and D. Zaitsev for their comments on a preliminary version of this paper. The authors are also grateful to an anonymous referee for helpful comments and suggestions. 


\section{Higher dimensional Riemann mapping theorems}

As already mentioned in the introduction, two bounded domains in $\mathbb{C}^{N}$ are almost never biholomorphically equivalent, even if they are diffeomorphic. A result which nicely illustrates this rather vague statement is the following. For any smoothly bounded strictly pseudoconvex domain $\Omega \subset \mathbb{C}^{N}$, there are arbitrarily small perturbations (in the $C^{\infty}$-topology) of this domain which are not biholomorphically equivalent to it (see Burns, Shnider, and Wells [BSW1978]; see also Greene and Krantz GK1982, GK1984). This fact also suggests that the local invariant geometry of the boundary of a domain plays an important role in determining its biholomorphic equivalence class. In this section, we shall present further evidence of this by giving results which show that under suitable conditions local biholomorphic equivalences of boundaries can yield global equivalence for domains.

A real-analytic hypersurface $M \subset \mathbb{C}^{N}$ is called spherical if for every $p \in M$ there exists a local biholomorphism $H:\left(\mathbb{C}^{N}, p\right) \rightarrow\left(\mathbb{C}^{N}, p^{\prime}\right)$, defined in a neighborhood of $p$, sending $M$ into the unit sphere $S^{2 N-1}$. (Here, $p^{\prime}$ is some point on $S^{2 N-1}$.) S.-S. Chern and S. Ji proved the following result which can be considered as a Riemann mapping theorem in higher dimensions.

Theorem 1.1 ([CJ1996]). Let $\Omega \subset \mathbb{C}^{N}$ be a bounded domain with connected, realanalytic, spherical boundary $\partial \Omega$. If $\Omega$ is simply connected, then $\Omega$ is biholomorphically equivalent to the unit ball $\mathbb{B}^{2 N} \subset \mathbb{C}^{N}$.

As a corollary, we obtain the following result, which characterizes those domains with real-analytic boundaries that are biholomorphically equivalent to the unit ball in terms of local geometric properties of their boundaries.

Theorem 1.2. Let $\Omega \subset \mathbb{C}^{N}$ be a bounded, simply connected domain with connected, real-analytic boundary $\partial \Omega$. Then $\Omega$ is biholomorphically equivalent to the unit ball $\mathbb{B}^{2 N} \subset \mathbb{C}^{N}$ if and only if $\partial \Omega$ is spherical.

Since the "only if" part of Theorem 1.2 is not explicitly stated in [CJ1996, we give here a sketch of a proof. It suffices to show that if $H$ is a biholomorphism from the ball $\mathbb{B}^{2 N}$ onto $\Omega$ (with $\Omega$ as in Theorem 1.2), then $H$ extends as a local biholomorphism across each point of the sphere $S^{2 N-1}$. First observe that, since $\mathbb{B}^{2 N}$ is pseudoconvex and $H$ is a biholomorphism onto $\Omega$, the latter is pseudoconvex (see e.g. Hörmander [Ho1990]). By a result of Bell [Bell1981] (see also more general results by Bell and Catlin [BC1982] and Diederich and Fornaess [DF1982]), it follows that $H$ extends as a smooth mapping from $S^{2 N-1}$ into $\partial \Omega$. Similarly, the inverse mapping $H^{-1}$ extends as a smooth mapping from $\partial \Omega$ into $S^{2 N-1}$. Hence, the boundary mapping is a diffeomorphism from $S^{2 N-1}$ onto $\partial \Omega$, and we deduce that $\Omega$ is strictly pseudoconvex. By the result of Lewy [Le1977] and Pinchuk [Pi1975] mentioned in the introduction, it follows that $H$ extends biholomorphically across each boundary point of $S^{2 N-1}$. The "only if" part of Theorem 1.2 follows.

The topological condition that $\Omega$ is simply connected is necessary for the conclusion of Theorem 1.1 to hold. Indeed, Burns and Shnider BS1976] showed that there are bounded domains with connected, real-analytic, spherical boundaries which are not biholomorphically equivalent to the ball $\mathbb{B}^{2 N}$. However, this topological restriction can be removed under the further assumption that the boundary of $\Omega$ is real algebraic. Recall that a connected, real-analytic hypersurface $M \subset \mathbb{C}^{N}$ is real algebraic if it is contained in the zero locus of a nontrivial real-valued polynomial. The following was proved by X. Huang and S. Ji. 
Theorem 1.3 ([HJ1998]). Let $\Omega \subset \mathbb{C}^{N}$ be a bounded domain with connected, real algebraic, spherical boundary $\partial \Omega$. Then $\Omega$ is biholomorphically equivalent to the unit ball $\mathbb{B}^{2 N} \subset \mathbb{C}^{N}$.

The conditions on the real algebraic domain $\Omega$ in the work [HJ1998] are stated in a slightly different but equivalent way; namely they assume no connectivity of $\partial \Omega$ but, instead, that the local biholomorphisms sending pieces of $\partial \Omega$ into the sphere $S^{2 N-1}$ send also the side of $\partial \Omega$ in $\Omega$ into the ball $\mathbb{B}^{2 N}$. The equivalence of Theorem 1.3 and the above statement rely on the fact that the boundary of a smoothly bounded pseudoconvex domain in $\mathbb{C}^{N}, N \geq 2$, is necessarily connected. (The latter fact also follows from the more general result given in Lemma 5.2 in [HJ1998.)

Recall that a holomorphic function $h(Z)$ is called algebraic if there is a nontrivial polynomial $P(Z, X)$, with $Z \in \mathbb{C}^{N}$ and $X \in \mathbb{C}$, such that $P(Z, h(Z)) \equiv 0$. A holomorphic mapping is called algebraic if its components are algebraic functions. One condition in Theorem 1.3 is that the real algebraic hypersurface $\partial \Omega$ is locally biholomorphic to the sphere $S^{2 N-1}$. One may ask if, under this condition, the local biholomorphism into the sphere can be chosen to be algebraic. Indeed, by a result of Webster We1977. (see §5), every such biholomorphism is necessarily algebraic. Hence, by a similar argument to the one above, we may transform Theorem 1.3 into the following. (We say that two domains in $\mathbb{C}^{N}$ are algebraically equivalent if they are biholomorphically equivalent by an algebraic mapping.)

Theorem 1.4. Let $\Omega \subset \mathbb{C}^{N}$ be a bounded domain with connected, real algebraic boundary $\partial \Omega$. Then $\Omega$ is algebraically equivalent to the unit ball $\mathbb{B}^{2 N} \subset \mathbb{C}^{N}$ if and only if $\partial \Omega$ is spherical.

Both Theorems 1.1 and 1.3 above rely on extension phenomena along paths of a local biholomorphic mapping sending one hypersurface into another. Previous results along these lines go back to Poincaré [P01907] for two dimensional spheres and Tanaka Ta1962 for spheres of higher dimension (see also Alexander A1974). Analogous extension results for ellipsoids were obtained by Webster [We1977], who classified ellipsoids in $\mathbb{C}^{N}$ up to biholomorphic equivalence. Extension of local biholomorphisms along paths contained in the source hypersurface in the strongly pseudoconvex case was considered by Pinchuk Pi1977] and Vitushkin, Ezhov, and Kruzhilin [VEK1985].

There is an extensive literature on other related global questions such as the structure of the automorphism groups of domains in $\mathbb{C}^{N}$ and boundary regularity of proper mappings between domains. We shall not address these questions here. We mention only the recent survey by Isaev and Krantz [K1999] dealing with the former topic. For the remainder of this survey, we shall restrict ourselves to local problems.

\section{LOCAL RESULTS FOR LEVI NONDEGENERATE HYPERSURFACES}

In this section, we shall recall some classical results on Levi nondegenerate hypersurfaces, due to Chern and Moser [CM1974] (see also earlier work by E. Cartan [Ca1932a], Ca1932b] and Tanaka [Ta1962], [Ta1967]), to motivate some of the studies presented in later sections. As mentioned above, we shall emphasize results that concern the structure of local mappings between such hypersurfaces.

Let $M \subset \mathbb{C}^{N}$ be a real hypersurface, and let $p_{0} \in M$. We shall write $n:=N-1$. Suppose that $M$ is defined near $p_{0}$ by the real equation $\rho(Z, \bar{Z})=0$, where $\rho$ is 
a smooth real-valued function with $\rho\left(p_{0}, \bar{p}_{0}\right)=0$ and $d \rho\left(p_{0}, \bar{p}_{0}\right) \neq 0$. Then $M$ is said to be Levi nondegenerate at $p_{0}$ if $J \rho\left(p_{0}, \bar{p}_{0}\right) \neq 0$, where $J$ denotes the complex Monge-Ampère type operator given by

$$
J u:=\operatorname{det}\left(\begin{array}{cc}
u & u_{Z} \\
u_{\bar{Z}}^{\tau} & u_{Z \bar{Z}}
\end{array}\right)
$$

here, $u_{Z}$ denotes the $1 \times N$ matrix $\left(\partial u / \partial Z_{j}\right)_{1 \leq j \leq N},{ }^{\tau}$ denotes transpose of a matrix, and $u_{Z \bar{Z}}$ denotes the $N \times N$ matrix $\left(\partial^{2} u / \partial Z_{j} \partial \bar{Z}_{k}\right)_{1 \leq j, k \leq N}$. The condition can also be given using the Levi form of $M$ which is defined as follows. For $p \in M$, the Levi form of $M$ at $p$ is the Hermitian form

$$
(\zeta, \eta) \mapsto \sum_{j, k=1}^{N} \frac{\partial^{2} \rho}{\partial Z_{j} \partial \bar{Z}_{k}}(p, \bar{p}) \zeta_{j} \bar{\eta}_{k}
$$

defined on the $n$-dimensional subspace $V_{p} \subset \mathbb{C}^{N}$,

$$
V_{p}:=\left\{\zeta \in \mathbb{C}^{N}: \sum_{j=1}^{N} \frac{\partial \rho}{\partial Z_{k}}(p, \bar{p}) \zeta_{k}=0\right\} .
$$

The Levi form of $M$ at $p$, as defined by (2.1), depends on the choice of holomorphic coordinates $Z$ and the choice of defining function $\rho$, but certain properties are invariant. For example, the number of nonzero eigenvalues is invariant, and so are the integers $m$ and $q$ if $m+q=n, m \geq q$, and $m$ and $q$ denote the numbers of nonzero eigenvalues which have the same sign. An equivalent definition of Levi nondegeneracy of $M$ at $p$ is that the Levi form of $M$ at $p$ is nondegenerate as a Hermitian form, i.e. has no zero eigenvalues.

Let us denote by $B$ the $n \times n$ Hermitian matrix representing the Levi form at $p_{0} \in M$ relative to some basis for $V_{p_{0}}$. (It is possible to choose this basis, replacing $\rho$ by $-\rho$ if necessary, so that $B$ is the diagonal matrix with $m$ ones followed by $q$ negative ones along the diagonal.) We denote by $S U(m+1, q+1)$ the group of unimodular $(N+1) \times(N+1)$ matrices that leave invariant the quadratic form

$$
\left(\left(\tilde{t}, \tilde{t}^{\prime}\right)\right):=t B t^{*}+\frac{i}{2}\left(t_{N} \bar{t}_{0}^{\prime}-t_{0} \bar{t}_{N}^{\prime}\right)
$$

where $\tilde{t}=\left(t_{0}, t, t_{N}\right), t=\left(t_{1}, \ldots, t_{n}\right)$ (with similar notation for $\tilde{t}^{\prime}$ and $t^{\prime}$ ), and * denotes Hermitian adjoint. Taking $\tilde{t}$ to be homogeneous coordinates in the complex projective space $\mathbb{C P}^{N}$, the equation $((\tilde{t}, \tilde{t}))=0$ defines a real quadric which we denote by $M_{0}$. The group $S U(m+1, q+1)$ acts on the quadric $M_{0}$. Furthermore, if $K \subset S U(m+1, q+1)$ denotes the finite subgroup consisting of the diagonal matrices with diagonal elements all equal to $\epsilon$ for some $(n+2)$-root of unity $\epsilon$, then the group $S U(m+1, q+1) / K$ acts on $M_{0}$ effectively; i.e. no element acts as the identity except the identity matrix. We denote by $G$ the isotropy subgroup of $S U(m+1, q+1) / K$, i.e. the maximal subgroup that preserves the origin in $\mathbb{C P}^{N}$.

In the nonhomogeneous coordinates $z=t / t_{0}, w=t_{N} / t_{0}$, the equation of the quadric $M_{0}$ takes the form

$$
\operatorname{Im} w=z B z^{*} .
$$


One can compute the isotropy group $G$ and show that the action of $G$ on $M_{0}$ is via the rational transformations $\left(z^{\prime}, w^{\prime}\right)=(f(z, w), g(z, w))$, where

$$
\begin{aligned}
& f(z, w)=\frac{\lambda(z+a w) U}{1-2 i z B a^{*}-\left(r+i a B a^{*}\right) w} \\
& g(z, w)=\frac{\sigma \lambda^{2} w}{1-2 i z B a^{*}-\left(r+i a B a^{*}\right) w} .
\end{aligned}
$$

Here, $\lambda>0, a=\left(a_{1}, \ldots, a_{n}\right) \in \mathbb{C}^{n}, r \in \mathbb{R}, \sigma= \pm 1$, and $U$ is an $n \times n$ matrix such that

$$
U B U^{*}=\sigma B
$$

It has been long known (see Poincaré Po1907, Tanaka Ta1962 for the case where $B$ is positive definite and Chern-Moser [CM1974 for the case of general nondegenerate $B$ ) that any germ of a biholomorphism $H$ that maps $M_{0}$ into itself and preserves the origin is of the form $H(z, w)=(f(z, w), g(z, w))$, where $f$ and $g$ are as in (2.5). Hence, we have identified the stability group $\operatorname{Aut}\left(M_{0}, 0\right)$ of the quadric $M_{0}$ at 0 with the isotropy subgroup $G \subset S U(p+1, q+1) / K$. By a slight abuse of notation, we also write $G$ for the group of rational transformations of the form (2.5); it should be clear from the context whether the elements of $G$ are considered as matrices or rational transformations.

Chern and Moser CM1974 give a normal form for a real-analytic hypersurface $M$ at a Levi nondegenerate point $p_{0}$, which can be described as follows. By applying a suitable affine transformation of $\mathbb{C}^{N}$ taking $p_{0}$ to the origin, we may assume, using the notation introduced above and denoting the new coordinates by $(z, w) \in \mathbb{C}^{n} \times \mathbb{C}$, that $M$ is given near $p_{0}=(0,0)$ by the equation

$$
\operatorname{Im} w=z B z^{*}+F(z, \bar{z}, \operatorname{Re} w),
$$

where $F(z, \bar{z}, s)$ is a real-valued, real-analytic function which is $O(3)$ in the weighted coordinate system in which $z$ and $\bar{z}$ have weight one and $s=\operatorname{Re} w$ has weight two. Denote by $\mathcal{F}$ the space of all such functions. It is shown in CM1974 that any biholomorphic transformation $H:\left(\mathbb{C}^{N}, 0\right) \rightarrow\left(\mathbb{C}^{N}, 0\right)$ preserving the form (2.7) of $M$, i.e. the image of $M$ is given by an equation of the form 2.7 with possibly another $F(z, \bar{z}, \operatorname{Re} w)$, occurs uniquely as a composition $H=T \circ R$, where $R \in G$ and $T(z, w)=(z+\tilde{f}(z, w), w+\tilde{g}(z, w))$ is a biholomorphic transformation such that $\tilde{f}$ is $O(2)$ in the weighted coordinate system where $z$ has weight one and $w$ weight two, $\tilde{g}$ is weighted $O(3)$, and

$$
\frac{\partial \tilde{f}}{\partial w}(0,0)=0, \quad \operatorname{Re} \frac{\partial^{2} g}{\partial w^{2}}(0,0)=0 .
$$

We denote the space of such transformations $T$ by $\mathcal{T}$. Consider the linear subspace $\mathcal{N} \subset \mathcal{F}$ defined as follows. First, decompose $F \in \mathcal{F}$ into $(k, l)$ type,

$$
F(z, \bar{z}, s)=\sum_{k, l=0}^{\infty} F_{k l}(z, \bar{z}, s),
$$

where each $F_{k l}(z, \bar{z}, s)$ is of type $(k, l)$, i.e. satisfies

$$
F_{k l}\left(t_{1} z, t_{2} \bar{z}, s\right)=t_{1}^{k} t_{2}^{l} F_{k l}(z, \bar{z}, s), \quad t_{1}, t_{2}>0 .
$$


Observe that the reality condition of $F$ is equivalent to $\overline{F_{k l}(z, \bar{z}, s)}=F_{l k}(z, \bar{z}, s)$. Denote by tr the contraction by the Hermitian form $\left(\zeta, \zeta^{\prime}\right) \rightarrow \zeta B \zeta^{\prime *}$; it operates on functions of type $(k, l)$ as a second order linear partial differential operator

$$
\operatorname{tr} F_{k l}=\frac{1}{k l}\left(\frac{\partial}{\partial z}\right) B\left(\frac{\partial}{\partial z}\right)^{*} F_{k l} .
$$

Then a function $N \in \mathcal{F}$ is in $\mathcal{N}$ if

$$
N(z, \bar{z}, s)=\sum_{\min (k, l) \geq 2} N_{k l}(z, \bar{z}, s)
$$

and, in addition, $\operatorname{tr} N_{22}=0, \operatorname{tr}^{2} N_{32}=0$, and $\operatorname{tr}^{3} N_{33}=0$. The Chern-Moser normal form can now be stated.

Theorem 2.13 (CM1974). Let $M \subset \mathbb{C}^{N}$ be a real-analytic hypersurface defined near 0 by an equation of the form (2.7) with nondegenerate $B$. Then for any rational transformation $R \in G$, there exists a unique biholomorphic transformation $H=T \circ R$, with $T \in \mathcal{T}$, such that $M$ is given near 0 in the coordinates $\left(z^{\prime}, w^{\prime}\right)=$ $H(z, w)$ by

$$
\operatorname{Im} w^{\prime}=z^{\prime} B z^{\prime *}+N\left(z^{\prime}, \bar{z}^{\prime}, \operatorname{Re} w^{\prime}\right)
$$

where $N \in \mathcal{N}$.

We shall say that $M$ is in Chern-Moser normal form if it is given by an equation as described in Theorem 2.13 .

As a consequence of Theorem 2.13, we may identify the stability group $\operatorname{Aut}(M, 0)$ of the Levi nondegenerate, real-analytic hypersurface $M$ given by (2.7) with a subset of the isotropy group $G \subset S U(m+1, q+1) / K$ as follows. Assume, as we may, that $M$ is in Chern-Moser normal form. Then for $H \in \operatorname{Aut}(M, 0)$ we factor $H$ as $H=T \circ R$, with $T \in \mathcal{T}$ and $R$ a rational mapping of the form 2.5, and identify $H \in \operatorname{Aut}(M, 0)$ with the matrix in $G$ corresponding to $R$. To see that the subset of $G$ corresponding to $\operatorname{Aut}(M, 0)$ is actually a subgroup, we shall use a result of Kruzhilin [Kr1985] stating that each mapping $T(z, w)=(z+\tilde{f}(z, w), w+\tilde{g}(z, w))$ occurring in the factorization $H=T \circ R$ of an element $H \in \operatorname{Aut}(M, 0)$ is such that $\tilde{f}$ vanishes to order at least three and $\tilde{g}$ to order at least four at 0 . (Here, the order of vanishing is the usual one and not the weighted order.) Thus, the derivatives of $H$ and those of $R$ up to second order agree at 0 . Since for a rational mapping $R=(f, g)$ of the form (2.5), the coefficients of the corresponding matrix in $G$ are determined by the system

$$
\frac{\partial f}{\partial z}(0)=\lambda U, \frac{\partial f}{\partial w}(0)=\lambda a U, \frac{\partial g}{\partial w}(0)=\sigma \lambda^{2}, \operatorname{Re} \frac{\partial^{2} g}{\partial w^{2}}(0)=2 \sigma \lambda^{2} r
$$

for $(\lambda, a, \sigma, r, U)$ as specified after (2.5), it follows that the identification of $\operatorname{Aut}(M, 0)$ with a subset of $G$ is in fact a group homomorphism, and, hence, we may think of $\operatorname{Aut}(M, 0)$ as a subgroup $G_{1}$ of $G$.

There is a canonical embedding of the group $G \subset S U(m+1, q+1) / K$ into the group of 2-jets of biholomorphic mappings $\left(\mathbb{C}^{N}, 0\right) \rightarrow\left(\mathbb{C}^{N}, 0\right)$ defined by taking a matrix in $G$ to the 2-jet of the rational mapping corresponding to it via (2.5). Since, by the result of $[\mathrm{Kr} 1985$ cited aboved, the 2-jet of $H \in \operatorname{Aut}(M, 0)$, for a realanalytic $M$ in Chern-Moser normal form, equals the 2-jet of the rational mapping $R$, where $H=T \circ R$ is the unique factorization of $H$ as described above, it follows in particular that any $H \in \operatorname{Aut}(M, 0)$ is completely determined by its 2-jet at 0 . (This 
will be discussed further below.) One may ask how the mapping $H \in \operatorname{Aut}(M, 0)$ depends on its 2-jet at 0 . For instance, do the 2-jets of mappings in $\operatorname{Aut}(M, 0)$ form a closed subgroup of the group of 2-jets? The answer is yes. Indeed, $G_{1}$ is a closed subgroup of $G$, which can be seen either by a careful inspection of the proof of Theorem 2.13 given in CM1974] or by using the geometric interpretation of the normal form given there. For the latter, the reader is referred to the original paper by CM1974 or to the survey article by Burns and Shnider [BS1977]. Another approach to these questions in a more general context will be given in $\S 4$.

The work of Chern and Moser has also been used to study in more detail the structure of the stability group of real hypersurfaces at Levi nondegenerate points. We shall only survey a couple of these results here since the main focus in this paper is not on Levi nondegenerate hypersurfaces.

If $M$ is given in the form (2.7) near $0 \in M$, then for $H=(f, g) \in \operatorname{Aut}(M, 0)$ we shall refer to the unique parameters $(\lambda, a, \sigma, r, U)$ obtained by solving (2.14) as the initial data of $H$. We have the following result by Beloshapka [Belo1979] and Loboda Lo1981, which implies that the stability group of a real-analytic hypersurface $M$ at a Levi nondegenerate point $p_{0}$ can be embedded into a smaller linear group than $G$, unless $M$ is locally biholomorphic at $p_{0}$ to a nondegenerate quadric.

Theorem 2.20 (Belo1979], [Lo1981]). Let $M \subset \mathbb{C}^{N}$ be a real-analytic hypersurface which is Levi nondegenerate at $0 \in M$ and which is given near 0 by an equation of the form (2.7). Assume that $M$ is not locally biholomorphic at 0 to the quadric (2.4). Then for any $H \in \operatorname{Aut}(M, 0)$ the parameters $\lambda, a, \sigma$ in its initial data are uniquely determined by the matrix $U$.

For strictly pseudoconvex hypersurfaces (i.e. those hypersurfaces whose Levi form is definite), more is known. Recall that a real-analytic hypersurface $M \subset \mathbb{C}^{N}$ is called spherical at a point $p_{0} \in M$ if it is locally biholomorphic to a piece of the sphere $S^{2 N-1}$. Kruzhilin and Loboda [KL1983] proved the following.

Theorem 2.21 ([KL1983]). Let $M \subset \mathbb{C}^{N}$ be a real-analytic hypersurface which is strictly pseudoconvex at $p_{0} \in M$. Assume that $M$ is not spherical at $p_{0}$. Then there exist coordinates $(z, w) \in \mathbb{C}^{n} \times \mathbb{C}$, with $N=n+1$, vanishing at $p_{0}$, such that $M$ is given in Chern-Moser normal form (see Theorem 2.13) and such that each $H \in \operatorname{Aut}(M, 0)$ is of the form

$$
H(z, w)=(f(z, w), g(z, w))=(U z, w),
$$

where $U$ is an $n \times n$ matrix which preserves the Levi form, i.e. such that $U B U^{*}=B$ with $B$ representing the Levi form as in Theorem 2.13.

There are many other local results for non-spherical hypersurfaces $M$ at strictly pseudoconvex points $p_{0} \in M$, such as e.g. estimates on a uniform neighborhood of extension for $H \in \operatorname{Aut}\left(M, p_{0}\right)$. We refer the interested reader to the survey article by Vitushkin V1985] for results in this direction. Let us also mention the work of Ezhov (see e.g. [Ez1986], [Ez1988]) on linearization of the stability group when the Levi form is nondegenerate but indefinite.

More recent work on normal forms includes that of Wong [Wo1982] and Barletta and Bedford [BB1990] on hypersurfaces in $\mathbb{C}^{2}$ at certain weakly pseudoconvex points. Stanton Sta1991] defined a different class of normal forms for rigid hypersurfaces in $\mathbb{C}^{2}$. The second author [Eb1998a], Eb1998b] considered a certain class of Levi degeneracies on hypersurfaces in higher dimensions. In recent work, Burns 
and X. Gong [BuG1999] obtain normal forms for Levi flat hypersurfaces at quadratic singularities. Ezhov and Schmalz studied certain Levi nondegenerate points on generic submanifolds of higher codimension (see e.g. [ES1997]).

The work of Chern and Moser (as well as that of E. Cartan and Tanaka) mentioned above also includes another, more intrinsic, approach to the local biholomorphic equivalence problem, which reduces this problem to that of an equivalence problem for systems of differential forms. The latter was solved by E. Cartan (see e.g. Sternberg Ste1983). We shall not describe this work in more detail here. We mention that the intrinsic approach was also used by the second author Eb1999 to study a class of everywhere Levi degenerate hypersurfaces in $\mathbb{C}^{3}$, and by Čap and Schichl [CS1997, Ezhov, Isaev, and Schmalz EIS1999, Schmalz and Slovak [ScS1999], Garrity and Mizner GM1997] to study certain Levi nondegenerate generic submanifolds of higher codimension.

\section{CR MANifolds, COMPlEX TANGENTS, AND NONDEGENERACY CONDITIONS}

We now turn to the study of a real submanifold $M \subset \mathbb{C}^{N}$ of codimension higher than (or equal to) one. Recall that a $(0,1)$-vector at a point $p$ in $\mathbb{C}^{N}$ is a complex tangent vector at $p$ of the form

$$
\left.\sum_{j=1}^{N} a_{j} \frac{\partial}{\partial \bar{Z}_{j}}\right|_{p}
$$

where $a_{j} \in \mathbb{C}$. A basic tool is the space of $(0,1)$-vectors in $\mathbb{C}^{N}$ at a point $p \in M$ tangent to $M$. This space is called the $C R$ (Cauchy-Riemann) tangent space of $M$ at $p$, and is denoted by $\mathcal{V}_{p}$. If the manifold $M$ is of (real) codimension $d \geq 1$, then

$$
N-d \leq \operatorname{dim}_{\mathbb{C}} \mathcal{V}_{p} \leq N-\frac{d}{2}
$$

Indeed, the lower bound follows from the fact that the dimension of the span of $\bar{\partial} \rho_{1}(p), \ldots, \bar{\partial} \rho_{d}(p)$ is less than or equal to $d$, where $\rho_{1}, \ldots, \rho_{d}$ are defining functions for $M$ near $p$ and $\bar{\partial}=\sum_{j=1}^{N}\left(\partial / \partial \bar{Z}_{j}\right) d \bar{Z}_{j}$. The upper bound in (3.1) is a consequence of the fact that $\mathcal{V}_{p} \cap \overline{\mathcal{V}}_{p}=\{0\}$, and $\mathcal{V}_{p} \oplus \overline{\mathcal{V}}_{p} \subset \mathbb{C} T_{p} M$. From (3.1), we see that if $d=1$, i.e. if $M$ is a real hypersurface, then $\operatorname{dim}_{\mathbb{C}} \mathcal{V}_{p}=N-1$ for every $p \in M$. For $d \geq 2$, the dimension of $\mathcal{V}_{p}$ can vary with $p$. We shall say that $M$ is $C R$ if $\operatorname{dim}_{\mathbb{C}} \mathcal{V}_{p}$ is constant over $M$. In this case the CR tangent spaces form a subbundle $\mathcal{V}$ of $\mathbb{C} T M$, called the $C R$ bundle of $M$. A smooth section of $\mathcal{V}$ is called a $C R$ vector field on $M$.

If $M$ is $\mathrm{CR}$, then the (complex) dimension of $\mathcal{V}_{p}$, for any $p \in M$, is called the $C R$ dimension of $M$. When the CR dimension of $M$ equals $N-d, M$ is called generic. Observe that when the $\mathrm{CR}$ dimension equals $N-d / 2$ (which in particular implies that $d$ is even), then $\mathcal{V}_{p} \oplus \overline{\mathcal{V}}_{p}=\mathbb{C} T_{p} M$. In this case, $M$ is in fact a complex submanifold of complex codimension $d / 2$. Another extreme occurs when the CR dimension of $M$ equals zero, in which case the manifold is said to be totally real.

We now extend, and give a more intrinsic definition of, the notion of Levi form introduced for hypersurfaces in $\mathbb{C}^{N}$ in $\S 2$. We say that a nonvanishing real 1-form $\theta$ on $M$ is a characteristic form if $\theta_{p}$ annihilates $\mathcal{V}_{p}$ (and hence also, by reality, annihilates $\overline{\mathcal{V}}_{p}$ ) for every $p \in M$. The Levi form $\mathcal{L}_{p}^{\theta}$, relative to $\theta$ at $p$, is the 
Hermitian form on $\mathcal{V}_{p}$ given by

$$
\mathcal{L}_{p}^{\theta}\left(X_{p}, Y_{p}\right):=\frac{1}{2 i}\left\langle\theta_{p},[X, \bar{Y}]_{p}\right\rangle
$$

where $X_{p}, Y_{p} \in \mathcal{V}_{p}, X, Y$ are $\mathrm{CR}$ vector fields on $M$ extending $X_{p}, Y_{p}$ respectively, $[\cdot, \cdot]$ denotes the commutator of vector fields, and $\langle\cdot, \cdot\rangle$ denotes the pairing between vectors and covectors. In the hypersurface case, we can relate the Levi form given by (2.1) to the one given by (3.2) in the following way. Take $\theta=-i \partial \rho$, and for $\zeta, \eta \in \mathcal{V}_{p}$ write

$$
X_{p}=\sum_{j=1}^{N} \bar{\zeta}_{j} \frac{\partial}{\partial \bar{Z}_{j}}, Y_{p}=\sum_{j=1}^{N} \bar{\eta}_{j} \frac{\partial}{\partial \bar{Z}_{j}} .
$$

Then, as can be verified using the identity $2\langle\theta,[X, \bar{Y}]\rangle=-\langle d \theta, X \wedge \bar{Y}\rangle$, the mapping (2.1) coincides with

$$
(\zeta, \eta) \mapsto \mathcal{L}_{p}^{\theta}\left(Y_{p}, X_{p}\right)
$$

3.1. Submanifolds with nondegenerate complex tangents. The study of real surfaces in $\mathbb{C}^{2}$, i.e. $M \subset \mathbb{C}^{2}$ with $\operatorname{dim}_{\mathbb{R}} M=2$, was begun by Bishop [Bi1965]. Observe, by (3.1), that for such an $M$ the dimension of $\mathcal{V}_{p}$ is either zero or one. Thus, if $M$ is $\mathrm{CR}$, then either it is totally real or a complex curve in $\mathbb{C}^{2}$. In particular, if $M$ is a real-analytic $\mathrm{CR}$ surface in $\mathbb{C}^{2}$, then $M$ is either locally biholomorphic to the real plane $\mathbb{R}^{2} \subset \mathbb{C}^{2}$ or the complex plane $\left\{Z_{2}=0\right\} \subset \mathbb{C}^{2}$. Hence, for a real-analytic surface $M$ in $\mathbb{C}^{2}$, the only interesting points on $M$, from the point of view of local biholomorphic equivalence, are those $p \in M$ where $\operatorname{dim} \mathcal{V}_{p}=1$ but $M$ is not $\mathrm{CR}$ in any neighborhood of $p$. Such points are called complex tangents of $M$. We first restrict our attention to a real-analytic surface $M \subset \mathbb{C}^{2}$, and a suitably nondegenerate complex tangent $p_{0}$ on this surface. It was shown Bi1965] that there are holomorphic local coordinates $(z, w) \in \mathbb{C}^{2}$, vanishing at $p_{0} \in M$, with $w=s+i t$ such that $M$ is given by an equation of the form

$$
s=z \bar{z}+\lambda\left(z^{2}+\bar{z}^{2}\right)+O\left(|z|^{3}\right), \quad t=O\left(|z|^{3}\right),
$$

where $0 \leq \lambda<\infty$. The constant $\lambda$ is a biholomorphic invariant, called the Bishop invariant. The complex tangent is said to be elliptic if $0 \leq \lambda<1 / 2$, parabolic if $\lambda=1 / 2$, and hyperbolic if $\lambda>1 / 2$. In the paper by Moser and Webster [MW1983, the authors obtain a normal form for an elliptic complex tangent with $0<\lambda<1 / 2$; more precisely, they show that there exists a biholomorphic transformation such that the equation for $M$ in the new holomorphic coordinates $(z, w), w=s+i t$, can be written

$$
s=z \bar{z}+\left(\lambda+\epsilon s^{\sigma}\right)\left(z^{2}+\bar{z}^{2}\right), \quad t=0,
$$

where $\lambda$ is the Bishop invariant, $\epsilon= \pm 1$ or $\epsilon=0$, and $\sigma \in \mathbb{Z}_{+}$. They also show that in the hyperbolic case, except for a discrete set of $\lambda$, there is a formal (not necessarily convergent) transformation to the form (3.1.2). In the hyperbolic case, one expects the normalization to diverge, and simple examples of algebraic hyperbolic surfaces which are formally equivalent to their normal forms (3.1.2) but not equivalent by a convergent transformation are given in [MW1983. Further work on divergence in transformations to normal forms was done by X. Gong [Go1994].

A consequence of (indeed, one of the motivations of) the work of Moser and Webster is that if $M \subset \mathbb{C}^{2}$ is a real-analytic surface with a complex elliptic tangent 
$p \in M$ with $0<\lambda<1 / 2$, then there exists a real-analytic one parameter family of disjoint embedded analytic disks with boundaries on $M$ and converging to $p$. The union of these disks $\widetilde{M} \subset \mathbb{C}^{2}$ forms a three dimensional real-analytic Levi flat 1 manifold-with-boundary with $M$ in its boundary. The manifold $\widetilde{M}$ coincides with the local holomorphic hull of $M$. The corresponding result in the case $\lambda=0$ was obtained by X. Huang and Krantz HK1995] (see also Moser M1985 for a special case of $\lambda=0$ ). For a merely smooth surface $M$ in $\mathbb{C}^{2}$, given locally near 0 by (3.1.1), the Bishop constant $\lambda$ is again a biholomorphic invariant of $M$. In this case, the existence of a smooth one parameter family of embedded analytic disks shrinking down to the elliptic complex tangent $0 \in M$ and the smoothness of the local holomorphic hull $\widetilde{M}$ was proved by Kenig and Webster KW1982.

In fact, the paper [MW1983] also contains a normal form for an $N$-manifold $M$ in $\mathbb{C}^{N}$ at a suitably nondegenerate elliptic tangent $p \in M$ with $0<\lambda<1 / 2$. (The Bishop invariant $\lambda$ for $M$ at $p$ is defined in an analogous way to the two dimensional case.) The description of the local hull of holomorphy $\widetilde{M}$ as a real-analytic Levi flat $(N+1)$-manifold-with-boundary follows as above. The study of the local hull $\widetilde{M}$, originally proposed by Bishop, of an $N$-manifold at a nondegenerate elliptic point was completed by X. Huang [Hu1998, who showed the real-analyticity of $\widetilde{M}$ when $M$ is real-analytic in the remaining case $\lambda=0$ as well as the smoothness of $\widetilde{M}$ when $M$ is merely smooth. The smooth case was based on previous partial results established by Kenig and Webster [KW1984. The reader is also referred to [Hu1998] for further information and references. The following theorem, which summarizes the results described above, give a complete solution to the question of Bishop regarding the local hull of holomorphy near an elliptic complex tangent.

Theorem 3.1.3. Let $M \subset \mathbb{C}^{N}$ be a smooth $N$-dimensional submanifold with an isolated elliptic complex tangent $p \in M(0 \leq \lambda<1 / 2)$. Then there exists a smooth family of disjoint embedded analytic disks with boundaries in $M$, converging to $p$, whose union $\widetilde{M}$ (the local holomorphic hull of $M$ near $p)$ is a smooth Levi flat $(N+$ 1)-dimensional manifold-with-boundary such that $M$ is contained in its boundary. Moreover, if $M$ is real-analytic, then $\widetilde{M}$ is also real-analytic.

A compact surface $M \subset \mathbb{C}^{2}$ is called a Bishop surface if all complex tangents are either elliptic or hyperbolic (and hence, in particular, isolated and nondegenerate). Bishop [Bi1965] showed that the number of elliptic complex tangents minus the number of hyperbolic ones equals the Euler characteristic number of a Bishop surface. A natural question is whether a Bishop surface $M$ is the boundary of a Levi flat three dimensional manifold-with-boundary $\widetilde{M}$. This is sometimes referred to as the complex Plateau problem. There is an abundant literature on this subject and a number of still open problems, but we will not address this further in this paper. We refer the reader for instance to Bedford and Gaveau BeG1983, Bedford and Klingenberg [BK1991], Kruzhilin [Kr1991], and also Gromov Gr1985] for related work.

We conclude this section by mentioning that normal forms for $m$-dimensional real-analytic submanifolds in $\mathbb{C}^{N}$, with $m<N$, at a suitably nondegenerate complex tangent have been studied by Beloshapka [Belo1997], and independently by Coffman [Co1999].

${ }^{1} \mathrm{~A}$ CR submanifold of $\mathbb{C}^{N}$ is called Levi flat if its Levi form at every point and relative to any characteristic form vanishes identically. 
3.2. Nondegeneracy conditions for generic submanifolds. For the remainder of this survey, we consider only CR submanifolds. In fact, we shall only consider generic submanifolds. This is not a serious restriction since any real-analytic CR submanifold $M$ of $\mathbb{C}^{N}$ is locally contained as a generic submanifold of a complex submanifold of $\mathbb{C}^{N}$; this complex submanifold is locally unique and is called the intrinsic complexification of $M$ (see [BER1999a]).

We shall focus in more detail on the questions raised in the introduction regarding the set of mappings (holomorphic or formal) between two generic submanifolds $M \subset \mathbb{C}^{N}$ and $M^{\prime} \subset \mathbb{C}^{N^{\prime}}$. One particular question is the following. Given two points $p_{0} \in M$ and $p_{0}^{\prime} \in M^{\prime}$, is the set of germs at $p_{0}$ of holomorphic mappings sending $M$ into $M^{\prime}$ and $p_{0}$ to $p_{0}^{\prime}$ determined by a finite number of parameters? Of particular interest is the case where $M=M^{\prime}, p_{0}=p_{0}^{\prime}$, and the mappings are all invertible. As mentioned in the introduction, the set of all such mappings is called the stability group of $M$ at $p_{0}$, denoted by $\operatorname{Aut}\left(M, p_{0}\right)$. Recall, from $\S 2$, that in the case of a Levi nondegenerate hypersurface this group is well understood: it can be identified with a finite dimensional Lie group, and hence depends on a finite number of parameters. We begin with an example showing that this is not the case in general.

Example 3.2.1. Let $M \subset \mathbb{C}^{3}$ be the hypersurface defined by

$$
\operatorname{Im} w=\left|z_{1}\right|^{2} .
$$

Here, the variables in $\mathbb{C}^{3}$ are taken to be $\left(z_{1}, z_{2}, w\right)$. We take $p_{0}=0$. Any holomorphic mapping of the form

$$
H\left(z_{1}, z_{2}, w\right)=\left(z_{1}, h\left(z_{1}, z_{2}, w\right), w\right)
$$

with $h$ an arbitrary holomorphic function vanishing at the origin, maps $(M, 0)$ into itself. Hence, the stability group $\operatorname{Aut}(M, 0)$ does not depend on finitely many parameters.

Observe that the equation (3.2.2) defining $M$ does not depend on the variable $z_{2}$. In particular, the holomorphic vector field $\partial / \partial z_{2}$ is tangent to $M$. A more subtle example is the following.

Example 3.2.4. Let $M \subset \mathbb{C}^{3}$ be the hypersurface defined by

$$
\operatorname{Im} w=\left|z_{1} z_{2}\right|^{2},
$$

and let $p_{0}=0$. Then, any holomorphic mapping of the form

$$
H\left(z_{1}, z_{2}, w\right)=\left(z_{1} e^{h\left(z_{1}, z_{2}, w\right)}, z_{2} e^{-h\left(z_{1}, z_{2}, w\right)}, w\right),
$$

with $h$ as in Example 3.2.1, is in $\operatorname{Aut}(M, 0)$. Hence, $\operatorname{Aut}(M, 0)$ again does not depend on finitely many parameters. Observe that the holomorphic vector field $z_{1} \partial / \partial z_{1}-z_{2} \partial / \partial z_{2}$ is tangent to $M$. However, there are no holomorphic coordinates at the origin in which the defining equation of $M$ is independent of one of the variables.

The previous two examples show the relevance of the following condition. A real-analytic generic submanifold $M \subset \mathbb{C}^{N}$ is called holomorphically degenerate at $p_{0} \in M$ if there exists a nontrivial germ at $p_{0}$ of a holomorphic vector field (with holomorphic coefficients) tangent to $M$ in a neighborhood of $p_{0}$. The relation between holomorphic degeneracy and biholomorphic self mappings of $M$ can be seen as follows. If $X$ is a nontrivial holomorphic vector field tangent to $M$, then 
the complex flow of $X$ is a one (complex) parameter family of biholomorphisms sending $M$ into itself. An infinite dimensional family of biholomorphic self mappings at $p_{0}$ may then be obtained by multiplying $X$ by arbitrary holomorphic functions vanishing at $p_{0}$.

The condition of holomorphic nondegeneracy was introduced by Stanton Sta1995, who showed that, for a real-analytic hypersurface $M$, holomorphic nondegeneracy at $p_{0}$ is equivalent to finite dimensionality of the space of infinitesimal CR automorphisms of $M$ at $p_{0}$. An interesting property is that for a connected realanalytic generic submanifold, holomorphic nondegeneracy at a point is equivalent to holomorphic nondegeneracy at every point (see [BER1999a]). For this reason, we shall often simply say that a connected real-analytic generic submanifold $M$ is holomorphically nondegenerate.

Another condition for finite determination is suggested by the following example.

Example 3.2.7. Let $M \subset \mathbb{C}^{3}$ be the generic submanifold given by

$$
\operatorname{Im} w_{1}=|z|^{2}, \quad \operatorname{Im} w_{2}=0,
$$

and let $p_{0}=0$. Here, the variables in $\mathbb{C}^{3}$ are denoted $\left(z, w_{1}, w_{2}\right)$. It is easy to check that $M$ is holomorphically nondegenerate. However, any holomorphic mapping of the form

$$
H\left(z, w_{1}, w_{2}\right)=\left(z, w_{1}, w_{2}+h\left(w_{2}\right)\right),
$$

where $h$ is a holomorphic function which vanishes to second order and has real Taylor coefficients at 0 , is in $\operatorname{Aut}(M, 0)$. The CR dimension of $M$ is one. All CR vector fields are multiples of $L=\partial / \partial \bar{z}-2 i z \partial / \partial \bar{w}_{1}$. The reader can verify that the vector space spanned at 0 by $L, \bar{L}$, and all repeated commutators of these two vector fields is three dimensional. Since the dimension of $M$ is four, this space is a proper subspace of $\mathbb{C} T_{0} M$.

A smooth generic submanifold $M \subset \mathbb{C}^{N}$ is said to be of finite type at $p_{0} \in M$ if the $\mathrm{CR}$ vector fields, their complex conjugates, and all repeated commutators of these vector fields at $p_{0}$ span $\mathbb{C} T_{p_{0}} M$. The hypersurfaces in Examples 3.2.1 and 3.2.4 are both of finite type at 0 , whereas the generic submanifold of codimension two in Example 3.2.7 is not. The notion of finite type was first introduced in this context by Kohn Ko1972 for hypersurfaces in $\mathbb{C}^{2}$, and for generic higher codimensional submanifolds by Bloom and Graham BlG1977.

We shall need to introduce two more nondegeneracy conditions for a generic real-analytic submanifold. Let $L_{1}, \ldots, L_{n}$ be a basis for the $\mathrm{CR}$ vector fields on $M$ near $p_{0}$; we write $N=n+d$, where $n$ is the CR dimension of $M$ and $d$ is the codimension. Let $\rho_{1}, \ldots, \rho_{d}$ be real-analytic defining functions for $M$ near $p_{0}$. We say that $M$ is finitely nondegenerate at $p_{0}$ if

$$
\operatorname{span} \mathbb{C}\left\{\left(L^{\alpha} \rho_{j Z}\right)\left(p_{0}, \bar{p}_{0}\right):|\alpha| \leq k, j=1, \ldots, d\right\}=\mathbb{C}^{N}
$$

for some $k \in \mathbb{Z}_{+}$. Here, we have used the notation $L^{\alpha}=L_{1}^{\alpha_{1}} \ldots L_{n}^{\alpha_{n}},|\alpha|=$ $\alpha_{1}+\ldots+\alpha_{n}$, and $f_{Z}=\partial f / \partial Z=\left(\partial f / \partial Z_{1}, \ldots, \partial f / \partial Z_{N}\right)$. If $k=k_{0}$ is the smallest integer for which (3.2.10) holds, then we also say that $M$ is $k_{0}$-nondegenerate at $p_{0}$. $M$ is 0-nondegenerate at $p_{0}$ if and only if $M$ is totally real at $p_{0}$. For a hypersurface, 1-nondegeneracy is equivalent to Levi nondegeneracy. In the previous examples, the first two (Examples 3.2.1 and 3.2.4) are not finitely nondegenerate, while Example 3.2.7 is 1-nondegenerate. 
Example 3.2.11. Let $M_{k l} \subset \mathbb{C}^{2}$ be the hypersurface

$$
\operatorname{Im} w=z^{k} \bar{z}^{l}+z^{l} \bar{z}^{k}
$$

where $k, l \in \mathbb{Z}_{+}$with $1 \leq l \leq k$. Then, $M_{k l}$ is finitely nondegenerate at 0 if and only if $l=1$, in which case it is $k$-nondegenerate.

The second nondegeneracy condition is that of essential finiteness, which was first defined in the work of the first author with Jacobowitz and Treves [BJT1985. As above, let $L_{1}, \ldots, L_{n}$ be a basis for the real-analytic $\mathrm{CR}$ vector fields on $M$ near $p_{0}$. We write

$$
L_{j}=\sum_{k=1}^{N} a_{j k}(Z, \bar{Z}) \frac{\partial}{\partial \bar{Z}_{k}}, \quad j=1, \ldots n,
$$

where the $a_{j k}(Z, \zeta)$ are holomorphic functions near $\left(p_{0}, \bar{p}_{0}\right) \in \mathbb{C}^{2 N}$. We set

$$
X_{j}:=\sum_{k=1}^{N} a_{j k}\left(p_{0}, \zeta\right) \frac{\partial}{\partial \zeta_{k}}, \quad j=1, \ldots n
$$

For $l=1, \ldots, d$ and $\alpha \in \mathbb{Z}_{+}^{n}$, we define

$$
c_{l \alpha}(W):=\left.X^{\alpha} \rho_{l}\left(p_{0}+W, \zeta\right)\right|_{\zeta=\bar{p}_{0}} .
$$

Then $c_{l \alpha}(0)=0$ for all $l=1, \ldots d$ and $\alpha \in \mathbb{Z}_{+}^{n}$. We say that $M$ is essentially finite at $p_{0}$ if $W=0$ is an isolated zero of the collection of functions $c_{l \alpha}(W)$, where $l=1, \ldots, d$ and $\alpha \in \mathbb{Z}_{+}^{n}$. The hypersurfaces in Examples 3.2.1 and 3.2.4 are not essentially finite at 0, whereas the generic submanifold in Example 3.2.7 is. Also, the hypersurfaces $M_{k l}$ in Example 3.2.11 are all essentially finite at 0. The following result (whose proof can be found in [BER1999a]) relates the three different notions of nondegeneracy given above.

Proposition 3.2.16. Let $M \subset \mathbb{C}^{N}$ be a connected real-analytic generic submanifold of codimension $d$ and $C R$ dimension $n$. Then the following conditions are equivalent.

(i) $M$ is holomorphically nondegenerate.

(ii) There exist $p_{1} \in M$ and $k>0$ such that $M$ is $k$-nondegenerate at $p_{1}$.

(iii) There exists an integer $\ell=\ell(M), 1 \leq \ell(M) \leq n$, such that the set of points in $M$ at which $M$ is not $\ell$-nondegenerate is a proper real-analytic subvariety of $M$. The number $\ell(M)$ is called the Levi number of $M$.

(iv) There exists $p_{2} \in M$ such that $M$ is essentially finite at $p_{2}$.

(v) The set of points in $M$ at which $M$ is not essentially finite is a proper real analytic subset of $M$.

More precisely, one can show that we have the following implications: $M$ is finitely nondegenerate at $p \Longrightarrow M$ is essentially finite at $p \Longrightarrow M$ is holomorphically nondegenerate at $p$. For a hypersurface $M$ in $\mathbb{C}^{N}, N>1$, we also have the implication: $M$ is essentially finite at $p \Longrightarrow M$ is of finite type at $p$. However, for higher codimensional generic manifolds there is no obvious relation between finite type and the notions of finite nondegeneracy and essential finiteness. A convenient sufficient condition for a generic submanifold $M$ to be essentially finite at a point $p_{0}$ is that it does not contain a germ of a nontrivial holomorphic subvariety through $p_{0}$. The latter condition is also referred to as being of D'Angelo finite type at $p_{0}$ (see D'Angelo [DA1982], DA1993]). For instance, it follows from a result of Diederich 
and Fornaess [DF1978 that a compact real-analytic submanifold in $\mathbb{C}^{N}$ does not contain any nontrivial holomorphic subvarieties, and hence such a submanifold is essentially finite at every point.

We now give an example showing that essential finiteness is strictly stronger than holomorphic nondegeneracy.

Example 3.2.17. Let $M \subset \mathbb{C}^{2}$ be the hypersurface given by

$$
\operatorname{Im} w=\operatorname{Re} w|z|^{2} .
$$

In this case, $M$ is holomorphically nondegenerate at every point, but is essentially finite only at points where $w \neq 0$.

We conclude this section by giving an example of a real-analytic hypersurface with Levi number $\ell(M)=2$. (In particular, $M$ is everywhere Levi degenerate but holomorphically nondegenerate by Proposition 3.2.16.)

Example 3.2.19. Let $M \subset \mathbb{C}^{3}$ be (the set of regular points of) the tube over the light cone, i.e. the real algebraic hypersurface defined by

$$
\left(\operatorname{Re} Z_{1}\right)^{2}+\left(\operatorname{Re} Z_{2}\right)^{2}-\left(\operatorname{Re} Z_{3}\right)^{2}=0,
$$

with $\left(\operatorname{Re} Z_{1}, \operatorname{Re} Z_{2}, \operatorname{Re} Z_{3}\right) \neq(0,0,0)$. It is easily verified that $M$ is 2 -nondegenerate (and hence also essentially finite) at every point. Moreover, through every point $p \in M$ there is a complex line which is contained in $M$.

\section{Structure of the Set of MAPpings BetWeEn GeneriC SubManifolds}

In this section, we shall show that under favorable conditions the set of mappings between two real-analytic generic submanifolds in $\mathbb{C}^{N}$ is given by finitely many parameters, and we shall describe this set more explicitly. Recall, from $\S 2$, that for a real-analytic hypersurface $M \subset \mathbb{C}^{N}$ the stability group $\operatorname{Aut}\left(M, p_{0}\right)$ at a Levi nondegenerate point $p_{0} \in M$ can be embedded as a closed subgroup of a certain finite dimensional linear group, which we also identified with the space of 2-jets of holomorphic mappings of $\mathbb{C}^{N}$.

To describe the set of mappings we need to introduce some notation. We denote by $E\left(\mathbb{C}^{N}, \mathbb{C}^{N}\right)_{\left(p, p^{\prime}\right)}$ the set of germs at $p$ of holomorphic mappings $H:\left(\mathbb{C}^{N}, p\right) \rightarrow$ $\left(\mathbb{C}^{N}, p^{\prime}\right)$ equipped with its usual inductive limit topology, and by $J^{k}\left(\mathbb{C}^{N}, \mathbb{C}^{N}\right)_{\left(p, p^{\prime}\right)}$ the space of $k$-jets of such. We also denote by

$$
j_{p}^{k}: E\left(\mathbb{C}^{N}, \mathbb{C}^{N}\right)_{\left(p, p^{\prime}\right)} \rightarrow J^{k}\left(\mathbb{C}^{N}, \mathbb{C}^{N}\right)_{\left(p, p^{\prime}\right)}
$$

the jet mapping of order $k$ which to each $H$ associates its $k$-jet $j_{p}^{k}(H)$, i.e. its Taylor polynomial at $p$ of degree $k$.

Let $M, M^{\prime} \subset \mathbb{C}^{N}$ be real-analytic generic submanifolds, and $p_{0} \in M, p_{0}^{\prime} \in$ $M^{\prime}$. We shall denote by $\mathcal{F}\left(M, p_{0}, M^{\prime}, p_{0}^{\prime}\right)$ the set of local biholomorphisms $H \in$ $E\left(\mathbb{C}^{N}, \mathbb{C}^{N}\right)_{\left(p_{0}, p_{0}^{\prime}\right)}$ such that $H(M) \subset M^{\prime}$. Observe that if $\left(M, p_{0}\right)$ and $\left(M^{\prime}, p_{0}^{\prime}\right)$ are not biholomorphically equivalent, then the set $\mathcal{F}\left(M, p_{0}, M^{\prime}, p_{0}^{\prime}\right)$ is empty (and vice versa). In this case, statements concerning $\mathcal{F}\left(M, p_{0}, M^{\prime}, p_{0}^{\prime}\right)$ below should be regarded as vacuous.

The following was proved by the authors of this survey.

Theorem 4.1 (BER1999b). Let $M, M^{\prime} \subset \mathbb{C}^{N}$ be real-analytic generic submanifolds of the same codimension $d$, and $p_{0} \in M, p_{0}^{\prime} \in M^{\prime}$. Assume that $M$ is of finite 
type and $k_{0}$-nondegenerate at $p_{0} \in M$, for some positive integer $k_{0}$. Then, the jet mapping

$$
j_{p_{0}}^{k_{0}(d+1)}: \mathcal{F}\left(M, p_{0}, M^{\prime}, p_{0}^{\prime}\right) \rightarrow J^{k_{0}(d+1)}\left(\mathbb{C}^{N}, \mathbb{C}^{N}\right)_{\left(p_{0}, p_{0}^{\prime}\right)}
$$

is a homeomorphism onto a real algebraic totally real submanifold of the jet space in (4.2).

The reader should observe that Theorem 4.1 implies in particular that, under the assumptions of that theorem, a mapping $H \in \mathcal{F}\left(M, p_{0}, M^{\prime}, p_{0}^{\prime}\right)$ is uniquely determined by finitely many parameters, namely its derivatives at $p_{0}$ up to order $k_{0}(d+1)$. It also contains the result that if a sequence of mappings $H^{l} \in \mathcal{F}\left(M, p_{0}, M^{\prime}, p_{0}^{\prime}\right)$ is such that the sequence of jets $j_{p_{0}}^{k_{0}(d+1)}\left(H^{l}\right)$ converges in the jet space, then the sequence of mappings $H^{l}$ converges to a mapping $H^{0} \in \mathcal{F}\left(M, p_{0}, M^{\prime}, p_{0}^{\prime}\right.$ ) (uniformly on a neighborhood of $p_{0}$ ).

Another corollary of Theorem 4.1 is that if $\left(M, p_{0}\right)$ satisfies the conditions in the theorem, then the stability group $\operatorname{Aut}\left(M, p_{0}\right)$, equipped with its natural topology, is a finite dimensional Lie group, isomorphic to a real algebraic subgroup of a jet space.

The results in BER1999b give additional information about the dependence of the mapping on its jet; namely, under the assumptions of Theorem 4.1, for every positive integer $l$ there are rational functions $R_{l}$ such that $j_{p_{0}}^{l}(H)=R_{l}\left(j_{p_{0}}^{k_{0}(d+1)}(H)\right)$ for any $H \in \mathcal{F}\left(M, p_{0}, M^{\prime}, p_{0}^{\prime}\right)$. We should point out that for the construction of the rational functions $R_{l},\left(M, p_{0}\right)$ and $\left(M^{\prime}, p_{0}^{\prime}\right)$ need not be biholomorphically equivalent. In fact, the construction also yields an infinite set of equations on the jet space such that $\left(M, p_{0}\right)$ and $\left(M^{\prime}, p_{0}^{\prime}\right)$ are biholomorphically equivalent if and only if there is a solution to this set of equations.

The examples given in $\S 3$ show that the conclusion of Theorem 4.1 fails to hold if either of the assumptions on $\left(M, p_{0}\right)$ is omitted. However, optimal conditions for embeddability of the stability group into some finite order jet space are not known.

As mentioned in $\S 2$, considerable work has been done in the Levi nondegenerate hypersurface case. In the case of higher codimension, there is a rather extensive literature on the automorphism group of nondegenerate quadrics. We mention here the papers by Tumanov and Henkin TH1983], Tumanov [Tu1988], Forstnerič [Fo1992, Sukhov [Su1994], Ezhov and Schmalz [ES1999]. We also mention the work of Beloshapka Belo1991] in which the stability group for higher codimensional generic submanifolds with suitably nondegenerate Levi forms was studied. Slightly weaker results than those of Theorem 4.1 were first obtained in the hypersurface case by the authors [BER1997. Later, Zaitsev [Z1997] showed that, under the assumptions of Theorem 4.1, Aut $\left(M, p_{0}\right)$ is a Lie group that can be embedded as a real-analytic submanifold in the jet space $J^{2 k_{0}(d+1)}\left(\mathbb{C}^{N}, \mathbb{C}^{N}\right)_{\left(p_{0}, p_{0}\right)}$. Related results for the infinitesimal CR automorphisms were obtained by Stanton [Sta1996], and also by the present authors [BER1998].

For the finite determination result mentioned immediately after Theorem 4.1, the assumption of finite nondegeneracy on $\left(M, p_{0}\right)$ can be weakened to that of essential finiteness. More precisely, the following result holds.

Theorem 4.3 ([BER1999c]). Let $M \subset \mathbb{C}^{N}$ be a real-analytic generic submanifold which is of finite type and essentially finite at $p_{0} \in M$. Then there exists a positive integer $K$ with the following property. If $M^{\prime} \subset \mathbb{C}^{N}$ is a real-analytic generic 
submanifold of the same dimension as $M$ and $p_{0}^{\prime} \in M^{\prime}$, then the jet mapping

$$
j_{p_{0}}^{K}: \mathcal{F}\left(M, p_{0}, M^{\prime}, p_{0}^{\prime}\right) \rightarrow J^{K}\left(\mathbb{C}^{N}, \mathbb{C}^{N}\right)_{\left(p_{0}, p_{0}^{\prime}\right)}
$$

is injective.

A precise description of the image of the mapping (4.4) (similar to the one given in Theorem 4.1) is not known in this case.

Results similar to those described in Theorems 4.1 and 4.3 hold for a more general class of holomorphic mappings than biholomorphisms. The reader is referred to BER1999b, BER1999c for more detailed information. These papers also contain results for merely smooth generic submanifolds and smooth CR mappings.

We conclude this section by pointing out that the set $\mathcal{F}\left(M, p_{0}, M^{\prime}, p_{0}^{\prime}\right)$ is in general empty; i.e. $\left(M, p_{0}\right)$ and $\left(M^{\prime}, p_{0}^{\prime}\right)$ are not biholomorphically equivalent, for $\left(M, p_{0}\right)$ and $\left(M^{\prime}, p_{0}^{\prime}\right)$ chosen at random. Convincing evidence of this is given by the following argument, which goes back to Poincaré [Po1907]. We shall, for simplicity, give the argument only in the case where $M$ and $M^{\prime}$ are real hypersurfaces. As in $\S 2$, we may choose local coordinates $(z, w) \in \mathbb{C}^{N-1} \times \mathbb{C}$ vanishing at $p_{0}$ so that $M$ is locally graphed near $p_{0}=(0,0)$ over the plane $\operatorname{Im} w=0$ by

$$
\operatorname{Im} w=\phi(z, \bar{z}, \operatorname{Re} w),
$$

where $\phi(0)=0$ and $d \phi(0)=0$. Denote by $T_{k}(z, \bar{z}, s)$ the Taylor polynomial of degree $k$ of the graphing function $\phi(z, \bar{z}, s)$. The number of Taylor coefficients in $T_{k}$, as $k \rightarrow \infty$, is asymptotically proportional to $k^{2 N-1}$. Now, the Taylor polynomials $T_{k}^{\prime}$ of degree $k$ similarly associated to all possible real hypersurfaces $M^{\prime} \subset \mathbb{C}^{N}$, graphed over the plane $\operatorname{Im} w^{\prime}=0$ at $p_{0}^{\prime}=(0,0)$, such that $\left(M^{\prime}, p_{0}^{\prime}\right)$ is biholomorphically equivalent to $\left(M, p_{0}\right)$ are obtained as follows. Substitute $z=f\left(z^{\prime}, w^{\prime}\right), w=g\left(z^{\prime}, w^{\prime}\right)$, where $H=(f, g)$ ranges over all possible biholomorphisms $\left(\mathbb{C}^{N}, 0\right) \rightarrow\left(\mathbb{C}^{N}, 0\right)$ whose Jacobian at $p_{0}$ maps the plane $\operatorname{Im} w=0$ to $\operatorname{Im} w^{\prime}=0$ (i.e. such that $\partial g / \partial z^{\prime}(0)=0$ ), in the equation (4.5) for $M$ to obtain

$$
\frac{g\left(z^{\prime}, w^{\prime}\right)-\overline{g\left(z^{\prime}, w^{\prime}\right)}}{2 i}=\phi\left(f\left(z^{\prime}, w^{\prime}\right), \overline{f\left(z^{\prime}, w^{\prime}\right)}, \frac{g\left(z^{\prime}, w^{\prime}\right)+\overline{g\left(z^{\prime}, w^{\prime}\right)}}{2}\right) .
$$

Then solve (4.6) for Im $w^{\prime}$ using the implicit function theorem. (This can be done, since $\partial g / \partial z^{\prime}(0)=0$, and hence $\partial g / \partial w^{\prime}(0) \neq 0$.) The resulting equation $\operatorname{Im} w^{\prime}=$ $\phi^{\prime}\left(z^{\prime}, \bar{z}^{\prime}, \operatorname{Re} w^{\prime}\right)$ describes the image $M^{\prime}$ as a graph over the plane $\operatorname{Im} w^{\prime}=0$, and $T_{k}^{\prime}\left(z^{\prime}, \bar{z}^{\prime}, s^{\prime}\right)$ is obtained by truncating the graphing function $\phi^{\prime}\left(z^{\prime}, \bar{z}^{\prime}, s^{\prime}\right)$ at level $k$. Counting the degrees of freedom in $T_{k}^{\prime}$ (by observing that only the Taylor polynomial of degree $k$ of the biholomorphism $H$, i.e. $j_{0}^{k}(H)$, affects $T_{k}^{\prime}$ ), we deduce that the Taylor coefficients of $T_{k}^{\prime}$ depend on a number of parameters which, as $k \rightarrow \infty$, are asymptotically proportional to $k^{N}$. Hence, by letting $k \rightarrow \infty$, we deduce that for a given real hypersurface $M \subset \mathbb{C}^{N}$ with $N \geq 2$, the "probability" that another real hypersurface $M^{\prime} \subset \mathbb{C}^{N}$ chosen at random is locally biholomorphic to $M$ is zero.

\section{Algebraicity of holomorphic MAPPings}

Recall that a connected real submanifold $M$ of $\mathbb{C}^{N}$ is called real algebraic if it is contained in an irreducible real algebraic subvariety of $\mathbb{C}^{N}$ of the same dimension as $M$. In particular, a real submanifold defined by real polynomial equations is real algebraic. In this section, we consider the following question. Let $M \subset \mathbb{C}^{N}$ and $M^{\prime} \subset \mathbb{C}^{N^{\prime}}$ be real algebraic generic submanifolds and $H$ a holomorphic mapping sending $M$ into $M^{\prime}$. Under what conditions is the mapping $H$ necessarily algebraic? 
Examples 3.2.1, 3.2.4, and 3.2.7, with $h$ suitably chosen, show that similar conditions to those imposed in $\S 3$ may be needed. Indeed, the authors showed the following (see also [BER1999a]).

Theorem 5.1 ([BER1996]). Let $M, M^{\prime} \subset \mathbb{C}^{N}$ be real algebraic generic submanifolds of the same dimension. Assume that $M$ is connected, of finite type at some point, and holomorphically nondegenerate. If $H$ is a biholomorphism defined in a neighborhood of some point on $M$, sending $M$ into $M^{\prime}$, then $H$ is algebraic.

It can be shown that the following two conditions for a connected real-analytic generic submanifold $M \subset \mathbb{C}^{N}$ are equivalent:

(a) $M$ has at least one point of finite type.

(b) Any holomorphic function defined on a neighborhood in $\mathbb{C}^{N}$ of a point in $M$ and whose trace on $M$ is real valued is necessarily constant.

In a different direction, Zaitsev proved the following result.

Theorem 5.2 ([BER1996]). Let $M \subset \mathbb{C}^{N}$ and $M^{\prime} \subset \mathbb{C}^{N^{\prime}}$ be real algebraic generic submanifolds. Assume that $M$ is connected and of finite type at some point, and that there is no nontrivial holomorphic subvariety contained in $M^{\prime}$. If $H$ is a holomorphic mapping defined in a neighborhood of some point on $M$, sending $M$ into $M^{\prime}$, then $H$ is algebraic.

In fact, the work of Zaitsev [Z1998] contains a more general and technical result from which both Theorems 5.1 and 5.2 may be derived. Results on partial algebraicity for mappings between real algebraic generic submanifolds were obtained by Coupet, Meylan, and Sukhov CMS1999, following earlier work of Sharipov and Sukhov ShS1996]. The first general theorem along the lines described in this section is due to Webster [We1977], who proved that a biholomorphic mapping between real algebraic Levi nondegenerate hypersurfaces in $\mathbb{C}^{N}$ is necessarily algebraic. Partial generalizations for hypersurfaces in different dimensions were obtained by X. Huang [Hu1994. Other results were recently obtained by Lamel La1999. We conclude this section by mentioning a recent preprint of Merker (http://xxx.lanl.gov/abs/math.CV/9906057), which states generalizations of Theorems 5.1 and 5.2 above.

\section{Convergence of formal Mappings}

We now consider a problem analogous to that considered in $\S 5$. There, we were given two real algebraic submanifolds of $\mathbb{C}^{N}$ and asked for conditions guaranteeing that a holomorphic mapping sending one into the other is necessarily algebraic. Here, we assume that the manifolds are real-analytic and consider formal mappings sending one into the other and ask for conditions implying that such a mapping is necessarily convergent.

The question of convergence is related to that of boundary regularity of holomorphic mappings. For instance, one might have two real-analytic hypersurfaces $M$ and $M^{\prime}$ and a holomorphic mapping defined on one side of $M$, extending smoothly to $M$ and sending $M$ into $M^{\prime}$. A reflection principle is a theorem asserting that such a mapping extends holomorphically across $M$. Although the question of convergence of formal mappings and that of the existence of a reflection principle seem quite similar, results concerning one problem do not necessarily imply results for the other. For instance, in one complex variable, by the Schwarz reflection principle, a holomorphic mapping defined in a neighborhood of the origin in the upper 
half plane, extending continuously up the real line and sending the real line into itself, extends holomorphically to a full neighborhood of the origin in the complex plane. However, any formal power series with real coefficients may be regarded as a formal mapping sending the real line into itself, but need not converge. Considerable work has been done on the reflection principle in higher dimensions, but we will not address this problem further in this survey. (For results and references the reader is referred to BER1999a.)

A formal mapping $H:\left(\mathbb{C}^{N}, 0\right) \rightarrow\left(\mathbb{C}^{N^{\prime}}, 0\right)$ is an $N^{\prime}$-vector $H=\left(H_{1}, \ldots, H_{N^{\prime}}\right)$ of formal power series in $Z=\left(Z_{1}, \ldots, Z_{N}\right)$ with vanishing constant term. When $N=N^{\prime}$, the formal mapping $H$ is said to be invertible if the Jacobian determinant of $H$ evaluated at 0 does not vanish. Let $M \subset \mathbb{C}^{N}$ and $M^{\prime} \subset \mathbb{C}^{N^{\prime}}$ be real-analytic submanifolds through the origin defined locally by the equations $\rho(Z, \bar{Z})=0$ and $\rho^{\prime}\left(Z^{\prime}, \bar{Z}^{\prime}\right)=0$, respectively, where $\rho=\left(\rho_{1}, \ldots, \rho_{d}\right)$ and $\rho^{\prime}=\left(\rho_{1}^{\prime}, \ldots, \rho_{d^{\prime}}^{\prime}\right)$. We say that a formal mapping $H$ sends $M$ into $M^{\prime}$ if

$$
\rho^{\prime}(H(Z), \bar{H}(\zeta))=a(Z, \zeta) \rho(Z, \zeta),
$$

where $a(Z, \zeta)$ is a $d^{\prime} \times d$ matrix of formal power series and where equality in (6.1) is understood in the sense of formal power series in $Z$ and $\zeta$. Observe that if $H$ is convergent, then (6.1) holds if and only if the holomorphic mapping $H$ sends $M$ into $M^{\prime}$.

Examples 3.2.1, 3.2.4, and 3.2.7 can be suitably modified to show that in general a formal mapping (even invertible) sending one real-analytic generic submanifold into another need not converge. However, the authors proved the following.

Theorem 6.2 ([BER1996] $)$. Let $M$ and $M^{\prime}$ be real-analytic hypersurfaces through the origin in $\mathbb{C}^{N}, N \geq 2$. Assume that neither $M$ nor $M^{\prime}$ contains a nontrivial holomorphic subvariety through 0 . Then any formal mapping $H:\left(\mathbb{C}^{N}, 0\right) \rightarrow\left(\mathbb{C}^{N}, 0\right)$ sending $M$ into $M^{\prime}$ is convergent.

If $M^{\prime} \subset \mathbb{C}^{N}$ is a real-analytic submanifold through the origin containing a positive dimensional holomorphic subvariety through 0 , then it is not hard to show that there is a nonconvergent formal mapping sending all of $\mathbb{C}^{N}$ into $M^{\prime}$. Hence, a consequence of Theorem 6.2 is the following characterization. A real-analytic hypersurface through the origin in $\mathbb{C}^{N}$ does not contain a nontrivial holomorphic subvariety through 0 if and only if all formal mappings $\left(\mathbb{C}^{N}, 0\right) \rightarrow\left(\mathbb{C}^{N}, 0\right)$ sending $M$ into itself are convergent.

For higher codimensional submanifolds, we have the following result.

Theorem 6.3 (BER1999c]). Let $M$ and $M^{\prime}$ be real-analytic generic submanifolds of the same dimension through the origin in $\mathbb{C}^{N}$. Assume that $M$ is of finite type at 0 , and that $M^{\prime}$ is essentially finite at 0 . Then any formal invertible mapping $H:\left(\mathbb{C}^{N}, 0\right) \rightarrow\left(\mathbb{C}^{N}, 0\right)$ sending $M$ into $M^{\prime}$ is convergent.

An interesting open question is whether the condition of invertibility of the formal mapping $H$ in Theorem 6.3 can be removed if the condition on $M^{\prime}$ is strengthened by assuming that $M^{\prime}$ does not contain a nontrivial holomorphic subvariety through 0 . This problem is open even in the case where $M$ and $M^{\prime}$ are real-analytic hypersurfaces. For instance, if $M^{\prime}$ does not contain a nontrivial holomorphic subvariety through 0 , but $M$ is merely of finite type at 0 , we do not know whether or not the conclusion of Theorem 6.2 still holds. 
As mentioned above, results on convergence of formal mappings do not imply a reflection principle in general. However, the implication does hold in the presence of a unique continuation principle for $\left(M, p_{0}\right)$ and $\left(M^{\prime}, p_{0}^{\prime}\right)$. We say that a unique continuation principle holds for $\left(M, p_{0}\right)$ and $\left(M^{\prime}, p_{0}^{\prime}\right)$, if for every pair of smooth germs at $p_{0}$ of CR mapping $22, g:\left(M, p_{0}\right) \rightarrow\left(M^{\prime}, p_{0}^{\prime}\right)$ such that $j_{p_{0}}^{k}(f)=j_{p_{0}}^{k}(g)$, for every positive integer $k$, it follows that $f \equiv g$. It is easy to see that unique continuation does not hold in general even when $\left(M, p_{0}\right)=\left(M^{\prime}, p_{0}^{\prime}\right)$ (cf. Examples $3.2 .1,3.2 .4$, and 3.2.7). An open question is to give sufficient conditions on $\left(M, p_{0}\right)$ and $\left(M^{\prime}, p_{0}^{\prime}\right)$ such that a unique continuation principle holds. In particular, we conjecture that if $M, M^{\prime} \subset \mathbb{C}^{N}$ are real-analytic hypersurfaces such that neither $M$ nor $M^{\prime}$ contains a nontrivial holomorphic subvariety through $p_{0} \in M$ or $p_{0}^{\prime} \in M^{\prime}$, then unique continuation holds. For $N=2$, the unique continuation principle conjectured above follows from the reflection principle proved by X. Huang [Hu1996]. In higher dimensions, the conjecture above is open, even under the stronger assumption that one of the mappings $f$ or $g$ is constant. Various related results under stronger conditions have been obtained by a number of mathematicians, but we shall not elaborate further on this question.

\section{Equivalence PROBlems}

We consider here the following questions. Let $M, M^{\prime} \subset \mathbb{C}^{N}$ be two real submanifolds of the same dimension, with distinguished points $p_{0} \in M$ and $p_{0}^{\prime} \in M^{\prime}$.

(a) Suppose $M$ and $M^{\prime}$ are real-analytic, and that $\left(M, p_{0}\right)$ and $\left(M^{\prime}, p_{0}^{\prime}\right)$ are formally equivalent; i.e. there is a formal invertible mapping $\left(\mathbb{C}^{N}, p_{0}\right) \rightarrow$ $\left(\mathbb{C}^{N}, p_{0}^{\prime}\right)$ sending $M$ into $M^{\prime}$. Are $\left(M, p_{0}\right)$ and $\left(M^{\prime}, p_{0}^{\prime}\right)$ biholomorphically equivalent?

(b) Suppose $M$ and $M^{\prime}$ are real algebraic, and that $\left(M, p_{0}\right)$ and $\left(M^{\prime}, p_{0}^{\prime}\right)$ are biholomorphically equivalent. Are they algebraically equivalent; i.e. is there an algebraic local biholomorphism sending $\left(M, p_{0}\right)$ into $\left(M^{\prime}, p_{0}^{\prime}\right)$ ?

(c) Suppose $M$ and $M^{\prime}$ are real-analytic CR submanifolds, and that $\left(M, p_{0}\right)$ and $\left(M^{\prime}, p_{0}^{\prime}\right)$ are $\mathrm{CR}$ equivalent; i.e. there is a smooth $\mathrm{CR}$ local diffeomorphism $\left(M, p_{0}\right) \rightarrow\left(M^{\prime}, p_{0}^{\prime}\right)$. Are $\left(M, p_{0}\right)$ and $\left(M^{\prime}, p_{0}^{\prime}\right)$ biholomorphically equivalent?

There are other interesting equivalence questions (e.g. $C^{k}$-equivalence) which we shall not mention further here. In this survey, we shall mainly focus on the first two questions (a) and (b) above. We shall, however, observe that if $M$ and $M^{\prime}$ are real-analytic CR submanifolds such that $\left(M, p_{0}\right)$ and $\left(M^{\prime}, p_{0}^{\prime}\right)$ are $\mathrm{CR}$ equivalent, then they are also formally equivalent. Indeed, the formal Taylor series of a CR function in a neighborhood of $p_{0}$ is necessarily the restriction to $M$ of a formal power series in $Z-p_{0}$ (see e.g. [BER1999a, Proposition 1.7.14]).

We begin by addressing question (a). Observe that the answer to that question is "no" in general, as is shown by the work of Moser and Webster (see §3). However, as a consequence of Theorem 6.3, we deduce that if two real-analytic generic submanifolds $M, M^{\prime} \subset \mathbb{C}^{N}$ of the same dimension, with $M$ of finite type at $p_{0} \in M$ and $M^{\prime}$ essentially finite at $p_{0}^{\prime} \in M^{\prime}$, are formally equivalent at $p_{0}$ and $p_{0}^{\prime}$, then they are also biholomorphically equivalent at those points. (In fact, Theorem 6.3 proves the stronger conclusion that every formal equivalence between $\left(M, p_{0}\right)$ and $\left(M^{\prime}, p_{0}^{\prime}\right)$ converges.) However, the authors know of no example of two real-analytic

2 A smooth mapping $f: M \rightarrow M^{\prime}$ between two smooth $\mathrm{CR}$ submanifolds is called $\mathrm{CR}$ if $f_{*}\left(\mathcal{V}_{p}\right) \subset \mathcal{V}_{f(p)}^{\prime}$ for every $p \in M$, where $\mathcal{V}$ and $\mathcal{V}^{\prime}$ are the CR bundles of $M$ and $M^{\prime}$ respectively. 
generic (or even CR) submanifolds which are formally equivalent but not biholomorphically equivalent. It seems reasonable to conjecture that formal equivalence of real-analytic CR submanifolds implies biholomorphic equivalence. The following result, due to the first and third author in collaboration with D. Zaitsev, supports this conjecture.

Theorem 7.1 ([BRZ1999] $)$. Let $M \subset \mathbb{C}^{N}$ be a real-analytic connected submanifold. Then there exists a proper real-analytic subvariety $V \subset M$ with the following property. For any $p_{0} \in M \backslash V$, any real-analytic submanifold $M^{\prime} \subset \mathbb{C}^{N}$ of the same dimension as that of $M$, and any $p_{0}^{\prime} \in M^{\prime}$, formal equivalence of $\left(M, p_{0}\right)$ and $\left(M^{\prime}, p_{0}^{\prime}\right)$ implies biholomorphic equivalence.

The real-analytic subvariety $V$ given by Theorem 7.1 is explicitly constructed in [BRZ1999] and in particular includes all points of $M$ which are not CR.

We now address question (b) above. The authors know of no pairs of real algebraic submanifolds $\left(M, p_{0}\right)$ and $\left(M^{\prime}, p_{0}^{\prime}\right)$ in $\mathbb{C}^{N}$ which are biholomorphically equivalent but not algebraically equivalent. A positive result in this direction is the following, which is implied by Theorem 5.1. Let $M, M^{\prime} \subset \mathbb{C}^{N}$ be real algebraic generic connected submanifolds of the same dimension, with $M$ of finite type at some point and $M^{\prime}$ holomorphically nondegenerate. Then, for any $p_{0} \in M$ and $p_{0}^{\prime} \in M^{\prime}$, if $\left(M, p_{0}\right)$ and $\left(M^{\prime}, p_{0}^{\prime}\right)$ are biholomorphically equivalent, they are also algebraically equivalent. The answer to question (b) for CR submanifolds in $\mathbb{C}^{2}$ is "yes". To show this, we observe that a (nontrivial proper) CR submanifold of $\mathbb{C}^{2}$ is of dimension one, two, or three. A real algebraic curve in $\mathbb{C}^{2}$ can be locally algebraically straightened to the $\left(\operatorname{Re} Z_{1}\right)$-axis by the (algebraic) implicit function theorem. A two dimensional CR submanifold $M$ is either totally real or complex. In the first case, $M$ can be locally algebraically flattened to $\mathbb{R}^{2} \subset \mathbb{C}^{2}$, and in the second case, to the complex line $\left\{Z_{1}=0\right\}$. In both cases, one applies the algebraic implicit function theorem. The crucial case is then that of two (three dimensional) hypersurfaces $M, M^{\prime} \subset \mathbb{C}^{2}$. For a connected real-analytic hypersurface in $\mathbb{C}^{2}$, the property of being holomorphically nondegenerate is equivalent to that of having a point of finite type (see [BER1999a, Chapter XI]). Thus, either the corollary to Theorem 5.1 mentioned above applies, or $M$ and $M^{\prime}$ can be locally algebraically flattened to $\left\{\operatorname{Im} Z_{2}=0\right\}$.

As in the case of question (b), the authors know of no examples giving a negative answer to question (c). This question is closely related to that of the existence of reflection principles as described in $\S 6$. We shall not pursue this topic further in this survey, but mention only that the problem is open even for real-analytic hypersurfaces in $\mathbb{C}^{2}$, and we conjecture that the answer is positive in this case.

We close this section by mentioning a problem related to the above questions. By the work of Moser and Webster, any real-analytic surface $M$ in $\mathbb{C}^{2}$ at an elliptic complex tangent $p_{0} \in M$, with $0<\lambda<1 / 2$ (where $\lambda$ denotes the Bishop invariant), is biholomorphically equivalent to a real algebraic one. The corresponding statement is false for real-analytic hypersurfaces in $\mathbb{C}^{2}$, as is proved below in the following example.

Example 7.2. Let $M$ be the real-analytic hypersurface through the origin in $\mathbb{C}^{2}$ defined by

$$
\operatorname{Im} w=\theta\left(\arctan |z|^{2}, \operatorname{Re} w\right)
$$


where $t=\theta(\xi, s)$ is the unique solution of the equation

$$
\xi\left(s^{2}+t^{2}\right)-t=0,
$$

with $\theta(0,0)=0$. We claim that $(M, 0)$ is not biholomorphically equivalent to any germ of a real algebraic hypersurface $\left(M^{\prime}, p_{0}^{\prime}\right)$ in $\mathbb{C}^{2}$. Indeed, there exists a smooth CR mapping $h: M \rightarrow \widetilde{M}$, where $\widetilde{M} \subset \mathbb{C}^{2}$ is given by

$$
\operatorname{Im} w=\operatorname{Re} w|z|^{2},
$$

such that $h$ vanishes to infinite order at 0 but the Jacobian of $h$ does not vanish identically in any neighborhood of 0 in $M$ (see Eb1996, and also BER1999a. Example 9.11.4]). If $(M, 0)$ were biholomorphically equivalent to $\left(M^{\prime}, p_{0}^{\prime}\right)$, where $M^{\prime} \subset \mathbb{C}^{2}$ is a real algebraic hypersurface, then by pulling the mapping $h$ back to $M^{\prime}$, we would obtain a smooth $\mathrm{CR}$ mapping from $M^{\prime}$ to $\widetilde{M}$ which vanishes to infinite order at the point $p_{0}^{\prime}$ but whose Jacobian does not vanish identically in any neighborhood of $p_{0}^{\prime}$ in $M^{\prime}$. Since $M^{\prime}$ and $\widetilde{M}$ are real algebraic hypersurfaces in $\mathbb{C}^{2}$ which are holomorphically nondegenerate, we may apply a result of the first and third authors, in a joint work with X. Huang [BHR1996], to conclude that such a mapping must be real-analytic. This, together with the nonvanishing of the Jacobian, contradicts the flatness of the pullback of $h$ at $p_{0}^{\prime}$.

This discussion and Example 7.2 lead to the interesting problem of deciding when a germ of a real-analytic submanifold $M$ at $p_{0}$ is biholomorphically equivalent to the germ of a real algebraic one. We know of no necessary or sufficient conditions for this even if $M$ is a real-analytic Levi nondegenerate hypersurface in $\mathbb{C}^{2}$. It would be interesting to find an explicit example of such which is not biholomorphically equivalent to a real algebraic one.

\section{Segre SETS And Finite type CONDition}

An important tool in many of the proofs of the results presented in $\S \S 4-6$ is the use of an invariant sequence of subsets of $\mathbb{C}^{N}$ attached to a germ of a real-analytic generic submanifold of $\mathbb{C}^{N}$. We shall describe these sets here, give some of their main properties, and allude to how they are used in the proofs of the results stated in the previous sections.

Let $M \subset \mathbb{C}^{N}$ be a generic real-analytic submanifold, with $0 \in M$, of codimension $d$ and CR dimension $n(N=n+d)$. Let $\rho(Z, \bar{Z})$, with $\rho=\left(\rho_{1}, \ldots, \rho_{d}\right)$, be a defining function for $M$ near 0 . For $U \subset \mathbb{C}^{N}$ a suitable small open neighborhood of 0 , we define the complexification $\mathcal{M} \subset U \times U^{*}$, where $U^{*}=\left\{\zeta \in \mathbb{C}^{N}: \bar{\zeta} \in U\right\}$, of $M$ to be the complex submanifold through the origin in $\mathbb{C}^{N} \times \mathbb{C}^{N}$ given by $\rho(Z, \zeta)=0$. For $p \in U$, we define the Segre variety of $p$, denoted $S_{1}(p, U)$ (which is also frequently denoted by $Q_{p}$ in the literature), to be the complex $n$ dimensional submanifold of $U$ defined by $\rho(Z, \bar{p})=0$. An important property of the Segre varieties is their invariance under holomorphic mappings; i.e. if $H:\left(\mathbb{C}^{N}, 0\right) \rightarrow\left(\mathbb{C}^{N}, 0\right)$ is a holomorphic mapping defined in a neighborhood of 0 and sending $M$ into another real-analytic generic submanifold $M^{\prime} \subset \mathbb{C}^{N^{\prime}}$ through 0 , then

$$
H\left(S_{1}(p, U)\right) \subset S_{1}^{\prime}\left(H(p), U^{\prime}\right),
$$

where $U^{\prime}$ is a suitable small open neighborhood of 0 in $\mathbb{C}^{N^{\prime}}$ and $S_{1}^{\prime}\left(p^{\prime}, U^{\prime}\right)$ denotes the Segre variety of $p^{\prime}$ (relative to $M^{\prime}$ ). The Segre varieties were introduced by Segre [Se1931], and their use in mapping problems for hypersurfaces pioneered 
by Webster ([We1977], We1978]). They have also been used by several authors in the study of normal forms and reflection principles (see e.g. Faran [Fa1980], Diederich-Webster [DW1980], Diederich-Fornaess [DF1988], Forstnerič [Fo1989], Huang [Hu1994], [Hu1996], Diederich-Pinchuk [DP1995], DP1998] and others).

For an integer $k \geq 2$, we define the $k$ th Segre set of $p$ in $U$ (relative to $M$ ) inductively by

$$
S_{k}(p, U)=\bigcup_{q \in S_{k-1}(p, U)} S_{1}(q, U)
$$

The reader should observe that $p \in M \cap U$ if and only if $p \in S_{1}(p, U)$. Hence, one can check that for $p \in M \cap U$, we have

$$
S_{1}(p, U) \subset S_{2}(p, U) \subset \ldots \subset S_{k}(p, U) \subset \ldots .
$$

An important property of the Segre sets is the fact that if $p \in M$ and $\zeta \in$ $S_{k-1}(p, U)^{*}$, then $(Z, \zeta) \in \mathcal{M}$ if and only if $Z \in S_{k}(p, U)$.

The $k$ th Segre set of $p \in M$ is in general not a submanifold (indeed, not even a holomorphic subvariety) but can be viewed as the image of an open neighborhood of the origin in $\mathbb{C}^{k n}$ under a holomorphic mapping $v^{k}:\left(\mathbb{C}^{k n}, 0\right) \rightarrow\left(\mathbb{C}^{N}, p\right)$, called the $k$ th Segre mapping. For this interpretation, the reader is referred to BER1999a, Chapter X]. The relevance of the Segre sets is illustrated by the following characterization of finite type.

Theorem 8.4 ([BER1996], BER1999a]). Let $M$ be a real-analytic generic submanifold of codimension d through the origin in $\mathbb{C}^{N}$. Then $M$ is of finite type at 0 if and only if there exists an integer $k \leq 2(d+1)$ such that $S_{k}(0, U)$ is a neighborhood of 0 in $\mathbb{C}^{N}$ for all sufficiently small neighborhoods $U$ of 0 .

The Segre sets have been used in conjunction with "reflection identities" for mappings. Rather than defining such in general, we illustrate this idea with a very simple example. Suppose that $M$ is a generic real-analytic submanifold through the origin in $\mathbb{C}^{N}$, and that $H:\left(\mathbb{C}^{N}, 0\right) \rightarrow(\mathbb{C}, 0)$ is a holomorphic mapping (function) defined in a neighborhood of 0 and sending $M$ into the real line $\left\{\operatorname{Im} Z^{\prime}=0\right\} \subset \mathbb{C}$. Hence, by complexification, we obtain the "reflection identity"

$$
H(Z)=\bar{H}(\zeta), \quad \forall(Z, \zeta) \in \mathcal{M}
$$

Putting $\zeta=0$ in (8.5), we deduce $H(Z)=\bar{H}(0)=H(0)$ for all $Z \in S_{1}(0, U)$. Hence, by taking $\zeta \in S_{1}(0, U)^{*}$ in (8.5), and using the important property of Segre sets mentioned above, we obtain $H(Z)=\bar{H}(\zeta)=H(0)$ for all $Z \in S_{2}(0, U)$. Inductively, we obtain, for every integer $k, H(Z)=H(0)$ for all $Z \in S_{k}(0, U)$. In particular, if $M$ is of finite type at 0 , then by Theorem 8.4 the mapping $H$ must be constant; i.e. there are no nonconstant holomorphic functions which are real valued on $M$. (This fact could have been proved directly using the CR vector fields and their conjugates, but the proof given here illustrates the simplest use of the Segre set interpretation of finite type combined with a reflection identity.)

As mentioned above, when the target submanifold $M^{\prime}$ is the real line in $\mathbb{C}$, the reflection identity (8.5) is immediate and takes a particularly simple form. In general, 
when $M^{\prime}$ is a real-analytic generic submanifold, satisfying suitable nondegeneracy conditions (i.e. finite nondegeneracy or, more generally, essential finiteness), similar but more complicated reflection identities involving also higher order derivatives of $H$ can be established (see e.g. [BER1999a, Chapters IX and XII]). The use of such reflection identities in conjunction with Theorem 8.4 above is a crucial part of the proofs of the results in $\S \S 4-6$.

\section{REFERENCES}

[A1974] Alexander, H., Holomorphic mappings from the ball and polydisc, Math. Ann. 209 (1974), 249-256. MR 50:5018

[BER1996] Baouendi, M. S., Ebenfelt, P., and Rothschild, L. P., Algebraicity of holomorphic mappings between real algebraic sets in $\mathbb{C}^{n}$, Acta Math. 177 (1996), 225-273. MR 99b:32024

[BER1997] — Parametrization of local biholomorphisms of real-analytic hypersurfaces, Asian J. Math. 1 (1997), 1-16. MR 99b:32022

[BER1998] CR automorphisms of real analytic manifolds in complex space, Comm. Anal. Geom. 6 (1998), 291-315. MR 99i:32024

[BER1999a] _ Real submanifolds in complex space and their mappings, Princeton University Press, Princeton Math. Ser. 47, Princeton, NJ, 1999. CMP 99:07

[BER1999b] Rational dependence of smooth and analytic CR mappings on their jets, Math. Ann. 315 (1999), 205-249.

[BER1999c] _ Convergence and finite determination of formal CR mappings, preprint http://xxx.lanl.gov/abs/math.CV/9904085.

[BHR1996] Baouendi, M. S., Huang, X., and Rothschild, L. P., [1], Regularity of CR mappings between algebraic hypersurfaces, Invent. Math. 125 (1996), 13-36. MR 97c:32028

[BJT1985] Baouendi, M. S., Jacobowitz, H., and Treves, F., On the analyticity of CR mappings, Ann. Math. 122 (1985), 365-400. [MR 87f:32044]

[BRZ1999] Baouendi, M. S., Rothschild, L. P., and Zaitsev, D., Equivalences of real submanifolds in complex space, preprint 2000.

[BB1990] Barletta, E. and Bedford, E., Existence of proper mappings from domains in $C^{2}$, Indiana Univ. Math. J. 39 (1990)), 315-338. MR 92b:32034

[BeG1983] Bedford, E. and Gaveau, B., Envelopes of holomorphy of certain 2-spheres, Amer. J. Math. 105 (1983), 975-1009. MR 84k:32016

[BK1991] Bedford, E. and Klingenberg, W., On the envelope of holomorphy of a 2-sphere in $C^{2}$, J. Amer. Math. Soc. 4 (1991), 623-646. MR 92j:32034

[BP1988] Bedford, E. and Pinchuk, S., Domains in $C^{2}$ with noncompact automorphism groups, Indiana Univ. Math. J. 47 (1998), 199-222. MR 99e:32058

[Bell1981] Bell, S., Biholomorphic mappings and the $\bar{\partial}-$ problem, Ann. of Math. 114 (1981), 103113. MR 82j:32039

[BC1982] Bell, S. and Catlin, D., Boundary regularity of holomorphic mappings, Duke Math. J. 49 (1982), 385-396. MR 84b:32037a

[Belo1979] Beloshapka, V. K., On the dimension of the group of automorphisms of an analytic hypersurface, (Russian), Izvestia Akad. Nauk SSSR, Ser. Mat. 43 (1979), 243-266, 479; English transl. in Math. USSR Izvestia.

[Belo1991] _ Holomorphic transformations of a quadric, (Russian), Mat. Sb. 182 (1991), 203-219. MR 92f:32065

[Belo1997] _ The normal form of the germ of a four-dimensional real manifold in $C^{5}$ at an $R C$-singular point in general position, (Russian), Mat. Zametki 61 (1997), 777-779; English transl. in Math. Notes. MR 99f:32012

[Bi1965] Bishop, E., Differentiable manifolds in complex Euclidean space, Duke Math. J. 32 (1965), 1-22. MR 34:369

[BlG1977] Bloom, T. and Graham, I., On "type" conditions for generic real submanifolds of $\mathbb{C}^{n}$, Invent. Math. 40 (1977), 217-243. MR 58:28644

[BuG1999] Burns, D. and Gong, X, Singular Levi-flat real analytic hypersurfaces, Amer. J. Math 121 (1999), 23-53. CMP 99:16

[BS1976] Burns, D. and Shnider, S., Spherical hypersurfaces in complex manifolds, Invent. Math. 33 (1976), 223-246. MR 54:7875 
[BS1977] — Real hypersurfaces in complex manifolds, Proceedings of Symposia in Pure Mathematics XXX, Part 2, Several Complex Variables, Amer. Math. Soc., Providence, RI, 1977, pp. 141-168. MR 56:8896

[BSW1978] Burns, D., Shnider, S., and Wells, R. O., Deformations of strictly pseudoconvex domains, Invent. Math. 46 (1978), 237-253. MR 58:1265

[CS1997] A. Čap and H. Schichl, Parabolic geometries and canonical Cartan connections, preprint 1997.

[Ca1932a] Cartan, E., Sur la géométrie pseudo-conforme des hypersurfaces de deux variables complexes, I, Ann. Math. Pura Appl. 11 (1932), 17-90, (or Euvres complètes. Partie II, Centre National de la Recherche Scientifique, Paris, 1984, 1231-1304). MR 85g:01032b

[Ca1932b] _ Sur la géométrie pseudo-conforme des hypersurfaces de deux variables complexes, II, Ann. Sc. Norm. Sup. Pisa 1 (1932), 333-354, (or Euvres complètes. Partie III, Centre National de la Recherche Scientifique, Paris, 1984, 1217-1238). MR 85g:01032d

[CJ1996] Chern, S.-S. and Ji, S., On the Riemann mapping theorem, Ann. of Math. 144 (1996), 421-439. MR 97m:32037

[CM1974] Chern, S.-S. and Moser, J. K., Real hypersurfaces in complex manifolds, Acta Math. 133 (1974), 219-271. MR 54:13112; MR 85a:32014

[CMS1999] Coupet, B., Meylan, F. and Sukhov, A., Holomorphic maps of algebraic CR manifolds, Internat. Math. Res. Notices (1999), 1-29. CMP 99:07

[Co1999] Coffman, A., Formal stability of the CR cross-cap, Pacific J. of Math. (1999) (to appear).

[DA1982] D'Angelo, J. P., Real hypersurfaces, orders of contact, and applications, Ann. of Math. 115 (1982), 615-637. MR 84a:32027

[DA1993] - Several complex variables and the geometry of real hypersurfaces, Studies in Advanced Mathematics, CRC Press, Boca Raton, FL, 1993. MR 94i:32022

[DF1978] Diederich, K. and Fornaess, J. E., Pseudoconvex domains with real-analytic boundary, Ann. of Math. 107 (1978), 371-384. MR 57:16696

[DF1982] _ Boundary behaviour of proper holomorphic mappings, Invent. Math. 67 (1982), 363-384. MR 85j:32039

[DF1988] - Proper holomorphic mappings between real-analytic pseudoconvex domains in $\mathbb{C}^{n}$, Math. Ann. 282 (1988), 681-700. MR 89m:32045

[DP1995] Diederich, K. and Pinchuk, S., Proper holomorphic maps in dimension 2 extend, Indiana Univ. Math. J. 44, 1089-1126. MR 97g:32031

[DP1998] _ Reflection principle in higher dimensions, Proceedings of the International Congress of Mathematicians, Vol. II, Berlin, 1998, pp. 703-712. CMP 99:02

[DW1980] Diederich, K. and Webster, S., A reflection principle for degenerate hypersurfaces, Duke Math. J. 47 (1980), 835-843. MR 82j:32046

[Eb1996] Ebenfelt, P., On the unique continuation problem for CR mappings into non-minimal hypersurfaces, J. Geom. Anal 6 (1996), 385-405. MR 98h:32030

[Eb1998a] , Normal forms and biholomorphic equivalence of real hypersurfaces in $\mathbb{C}^{3}$, Indiana Univ. Math. J. 42 (1998), 311-366. CMP 99:03

[Eb1998b] - New invariant tensors in CR structures and a normal form for real hypersurfaces at a generic Levi degeneracy, J. Differential Geom. 50 (1998), 207-247. CMP 99:11

[Eb1999] _ Uniformly Levi degenerate CR manifolds; the 5 dimensional case, http://xxx.lanl.gov/abs/math.CV/9905163, 1999.

[Ez1986] Ezhov, V. V., Linearization of the stability group of a class of hypersurfaces, (Russian), Uspekhi Mat. Nauk 41 (1986), 203-204; English transl. in Russian Math. Surveys. MR 87k:32033

[Ez1988] — An example of a real-analytic hypersurface with a nonlinearizable stability group, (Russian), Mat. Zametki 44 (1988), 824-828; English transl. in Math. Notes. MR 90e:32020

[EIS1999] Ezhov, V. V., Isaev, A. V. and Schmalz, G., Invariants of elliptic and hyperbolic CRstructures of codimension 2, Internat. J. Math. 10 (1999), 1-52. CMP 99:10

[ES1997] Ezhov, V. V. and Schmalz, G., Normal form and two-dimensional chains of an elliptic CR manifold in $\mathbf{C}^{4}$, J. Geom. Anal. 6 (1997), 495-529. MR 98m:32025

[ES1999] _ Holomorphic automorphisms of nondegenerate CR quadrics: An explicit description, J. Geom. Anal. (to appear).

[Fa1980] Faran, J. J., Segre families and real hypersurfaces, Invent. Math. 60 (1980), 135-172. MR 81i:32018 
[Fe1974] Fefferman, C., The Bergman kernel and biholomorphic mappings of pseudoconvex domains, Invent. Math. 26 (1974), 1-65. MR 50:2562

[Fo1989] Forstnerič, F., Extending proper holomorphic mappings of positive codimension, Invent. Math. 95 (1989), 31-62. MR 89j:32033

[Fo1992] , Mappings of quadric Cauchy-Riemann manifolds, Math. Ann. 292 (1992), 163180. MR 93e:32033

[GM1997] Garrity, T. and Mizner, R., The equivalence problem for higher-codimensional CR structures, Pacific J. Math. 177 (1997), 211-235. MR 99b:32007

[Go1994] Gong, X., Divergence for the normalization of real analytic glancing hypersurfaces, Comm. Partial Differential Equations 19 (1994), 643-654. MR 95f:58079

[GK1982] Greene, R. E. and Krantz, S. G., Deformation of complex structures, estimates for the $\bar{\partial}$ equation, and stability of the Bergman kernel, Adv. Math. 43 (1982), 1-86. MR 84b:32026

[GK1984] _ Stability of the Carathéodory and Kobayashi metrics and applications to biholomorphic mappings, Complex analysis of several variables (Madison, WI, 1982), Proc. Symp. Pure Math., 41, Amer. Math. Soc., Providence, RI, 1984. MR 85k:32043

[Gr1985] Gromov, M., Pseudoholomorphic curves in symplectic manifolds, Invent. Math. 82 (1985), 307-347. MR 87j:53053

[Ho1990] Hörmander, L., An Introduction to Complex Analysis in Several Variables. Third edition, North-Holland Publishing Co., Amsterdam-New York, 1990. MR 91a:32001

[Hu1994] Huang, X., On the mapping problem for algebraic real hypersurfaces in the complex spaces of different dimensions, Ann. Inst. Fourier (Grenoble) 44 (1994), 433-463. MR 95i:32030

[Hu1996] _ Schwarz reflection principle in complex spaces of dimension two, Comm. Partial Diff. Eq. 21 (1996), 1781-1828. MR 97m:32043

[Hu1998] _ On an n-manifold in $C^{n}$ near an elliptic complex tangent, J. Amer. Math. Soc. 11 (1998), 669-692. MR 98m:32026

[HJ1998] Huang, X. and Ji, S., Global holomorphic extension of a local map and a Riemann mapping theorem for algebraic domains, Math. Res. Lett. 5 (1998), 247-260. MR 99d:32013

[HK1995] Huang, X. and Krantz, S. G., On a problem of Moser, Duke Math. J. 78 (1995), 213-228. MR 96f:32026

[IK1999] Isaev, A. V. and Krantz, S. G., Domains with non-compact automorphism group: A survey, Adv. Math. 146 (1999), 1-38. CMP 2000:01

[Ke1912] Kellogg, O. D., Harmonic functions and Green's integral, Trans. Amer. Math. Soc. 13 (1912), 109-132.

[KW1982] Kenig, C. E. and Webster, S. M., The local hull of holomorphy of a surface in the space of two complex variables, Invent. Math. 67 (1982), 1-21. MR 84c:32014

[KW1984] , On the hull of holomorphy of an n-manifold in $C^{n}$, Ann. Scuola Norm. Sup. Pisa 11 (1984), 261-280. MR 86d:32019

[Ko1972] Kohn, J. J., Boundary behaviour of $\delta$ on weakly pseudo-convex manifolds of dimension two, J. Differential Geom. 6 (1972), 523-542. MR 48:727

[Kr1985] Kruzhilin, N. G., Local automorphisms and mappings of smooth strictly pseudoconvex hypersurfaces, (Russian), Izv. Akad. Nauk SSSR Ser. Mat. 49 (1985), 566-591. MR 86j:32046

[Kr1991] _ Two-dimensional spheres on the boundaries of pseudoconvex domains in $\mathbb{C}^{2}$, (Russian), Izv. Akad. Nauk SSSR Ser. Mat. 55 (1991), 1194-1237. MR 93e:32017

[KL1983] Kruzhilin, N. G. and Loboda A. V., Linearization of local automorphisms of pseudoconvex surfaces, (Russian), Dokl. Akad. Nauk SSSR 271 (1983), 280-282; English transl. in Soviet Math. Dokl. 28 (1983), 70-72. MR 85c:32052

[La1999] Lamel, B., Holomorphic maps of real submanifolds in complex spaces of different dimensions, preprint, 1999.

[Le1977] Lewy, H., On the boundary behavior of holomorphic mappings, Acad. Naz. Lincei 35 (1977), 1-8.

[Lo1981] Loboda, A. V., On local automorphisms of real-analytic hypersurfaces, (Russian), Izvestia Akad. Nauk SSSR, Ser. Mat. 45 (1981), 620-645; English transl. in Math. USSR Izvestia 18 (1982), 537-559. MR 83k:32017]

[M1985] Moser, J., Analytic surfaces in $C^{2}$ and their local hull of holomorphy, Ann. Acad. Sci. Fenn. Ser. A I Math. 10 (1985), 397-410. MR 87c:32024 
[MW1983] Moser, J. and Webster, S. M., Normal forms for real surfaces in $\mathbb{C}^{2}$ near complex tangents and hyperbolic surface transformations, Acta Math. 150 (1983), 255-296. MR 85c:32034

[Pi1975] Pinchuk, S. I., The analytic continuation of holomorphic mappings, (Russian), Mat. Sb. (N.S.), 98 (140), 1975, 416-435; English transl. in. Math. USSR Sbornik 27 (1975), 375-392. MR 52:14371

[Pi1977] Pinchuk, S. I., Analytic continuation of mappings along strictly pseudo-convex hypersurfaces, (Russian), Dokl. Akad. Nauk SSSR 236 (1977), 544-547.

[Po1907] Poincaré, H., Les fonctions analytiques de deux variables et la représentation conforme, Rend. Circ. Mat. Palermo, II. Ser. 23 (1907), 185-220.

[ScS1999] Schmalz, G. and Slovak, J., Geometry of hyperbolic and elliptic CR-manifolds of codimension two, http://xxx.lanl.gov/abs/math.DG/9903046, 1999.

[Se1931] Segre, B., Intorno al problem di Poincaré della rappresentazione pseudo-conform, Rend. Acc. Lincei 13 (1931), 676-683.

[ShS1996] Sharipov, R. and Sukhov, A., On CR-mappings between algebraic Cauchy-Riemann manifolds and separate algebraicity for holomorphic functions, Trans. Amer. Math. Soc. 348 (1996), 767-780. MR 96g:32019

[Sta1991] Stanton, N., A normal form for rigid hypersurfaces in $C^{2}$, Amer. J. Math. 113 (1991), 877-910. MR 92k:32031

[Sta1995] _ Infinitesimal CR automorphisms of rigid hypersurfaces, Amer. J. Math. 117 (1995), 141-167. MR 96a:32036

[Sta1996] _, Infinitesimal CR automorphisms, Amer. J. Math. 118 (1996), 209-233. MR 97h:32027

[Ste1983] Sternberg, S., Lectures on Differential Geometry, Chelsea Publ. Co., New York, NY, 1983. MR 88f:58001

[Su1994] Sukhov, A., On CR mappings of real quadric manifolds, Michigan Math. J. 41 (1994), 143-150. MR 95h:32006

[Ta1962] Tanaka, N., On the pseudo-conformal geometry of hypersurfaces of the space of $n$ complex variables, J. Math. Soc. Japan 14 (1962), 397-429. MR 26:3086

[Ta1967] _ On generalized graded Lie algebras and geometric structures. I, J. Math. Soc. Japan 19 (1967), 215-254, (erratum 36, p. 1568). MR 36:4470

[Tu1988] Tumanov, A. E., Finite-dimensionality of the group of CR automorphisms of a standard CR manifold, and proper holomorphic mappings of Siegel domains, (Russian), Izvestia Akad. Nauk SSSR, Ser. Mat. 52 (1988), 651-659, 672; English transl. in Math. USSR Izvestia.

[TH1983] Tumanov, A. E., Henkin, G. M., Local characterization of holomorphic automorphisms of Siegel domains, (Russian), Funktsional. Anal. i Prilozhen. 17 (1983), 285-294; English transl. in Functional Anal. Appl. MR 86a:32063

[V1985] Vitushkin, A. G., Holomorphic mappings and the geometry of hypersurfaces, Encyclopaedia of Mathematical Sciences, Vol. 7, Several Complex Variables I, Springer-Verlag, Berlin, 1985, pp. 159-214.

[VEK1985] Vitushkin, A. G., Ezhov, V. V., and Kruzhilin, N. G., Continuation of holomorphic mappings along real-analytic hypersurfaces, Current problems in mathematics. Mathematical analysis, algebra, topology, Trudy Mat. Inst. Steklov. 167 (1985), 60-95. MR 87d:32039

[We1977] Webster, S. M., On the mapping problem for algebraic real hypersurfaces, Invent. Math. 43 (1977), 53-68. MR 57:431

[We1978] _ On the reflection principle in several complex variables, Proc. Amer. Math. Soc. 71 (1978), 26-28. MR 57:16681

[Wo1982] Wong. P. P., A construction of normal forms for weakly pseudoconvex CR manifolds in $\mathbb{C}^{2}$, Invent. Math. 69 (1982), 311-329, (Erratum: 71 (1983), p. 241). MR 84e:32020a MR 84e:32020b

[Z1997] Zaitsev, D., Germs of local automorphisms of real-analytic CR structures and analytic dependence on $k$-jets, Math. Research Lett. 4 (1997), 823-842. MR 99a:32007

[Z1998] - Algebraicity of local holomorphisms between real-algebraic submanifolds of complex spaces, preprint 1998. 
Department of Mathematics, University of California at San Diego, La Jolla, CA 92093

E-mail address: sbaouendi@ucsd.edu

Department of Mathematics, Royal Institute of Technology, 10044 Stockholm, SweDEN

E-mail address: ebenfelt@math.kth.se

Department of Mathematics, University of California at San Diego, La Jolla, Ca 92093

E-mail address: lrothschild@ucsd.edu 